\title{
Flexible organic crystals. Understanding the tractable co- existence of elastic and plastic bending
}

\author{
Indira S. Divya, ${ }^{1,2}$ Saravanan Kandasamy, ${ }^{3}$ Krzysztof Woźniak ${ }^{3 *}$ and Sunil Varughese ${ }^{1,2 *}$ \\ ${ }^{1}$ Chemical Science and Technology Division, CSIR-National Institute for Interdisciplinary Science and \\ Technology, Trivandrum 695019, India \\ ${ }^{2}$ Academy of Scientific and Innovative Research (AcSIR), Ghaziabad 201002, India \\ ${ }^{3}$ Crystallochemistry Laboratory, University of Warsaw, Warsaw 02-093, Poland \\ E-mail: s.varughese@ niist.res.in; kwozniak@chem.uw.edu.pl; Phone: +91 (0)471 2515438
}

\begin{abstract}
As an emerging class of flexible materials, mechanically bendable molecular crystals are broadly classified as either elastic or plastic. Nevertheless, flexible organic crystals having elasticity and plasticity coexisting under different stress settings are exceptional; hence, it is imperative to establish the concurring factors that beget this rare occurrence. We report a series of halogen-substituted benzil crystals showing elastic bending (within 2.45\% strain), followed by elastoplastic deformation at ambient conditions. At higher stress settings, they display exceptional plastic flexibility that one could bend, twist, or even coil them around a capillary tube. Structural, computational, and microscopy studies reveal the microscopic and macroscopic basis for the exciting coexistence of elastic, elastoplastic, and plastic properties in the crystals. The isotropic distribution of weak interactions and the dispersive interactions that stitch the interlayer region enables the system to adopt partial molecular movements, restoring the original state within the elastic strain. The novel polymorph of F-derivative (BZF) shows exceptional mechanical deformation characteristics vis-à-vis the brittle nature of the known form (BZF_O). Comparative studies with oxalate compounds, wherein the twisted diketo moiety in benzil replaced with a rigid and coplanar central oxalate moiety, enabled us to understand the effect of the anisotropic factor in the crystal packing induced by the $\mathrm{C}=\mathrm{O} \cdots \mathrm{C}$ tetral interactions. The enhanced anisotropy reduced the elastic regime, making the oxalate crystals more prone to plastic deformation. The work highlights the possible coexistence of orthogonal mechanical characteristics in molecular crystals and further construed the concurrent effect of the microscopic and macroscopic elements in attaining this exceptional mechanical trait.
\end{abstract}




\section{Introduction}

Organic crystalline materials attract immense academic and industrial stakes because of their exciting physicochemical properties and unique utilities in an ample repertoire of applications such as optoelectronics, ${ }^{1,2}$ mechanical actuators, ${ }^{3,4}$ pharmaceuticals, ${ }^{5,6}$ and explosives. ${ }^{7,8}$ From the practical application perspective, the materials need to attain excellent workability and in this regard mechanical deformability (plastic or elastic) is indispensable. ${ }^{9,10}$ The mechanically inflexible and brittle attributes of molecular crystalline materials on the macroscopic level au contraire limits their utility; it makes them far from the stage of materials development compared to flexible polymers exhibiting viscoelasticity. ${ }^{11,12}$ Not surprisingly, immense interest has been attached to comprehending the mechanical properties in molecular materials in recent times. ${ }^{13-17}$ Momentous progress attained in this field owes much to the systematic and earnest efforts made to gain significant insights into precise mechanisms that govern the structural and mechanical properties of molecular materials. ${ }^{18}$ Such understanding is imperative to attain predictable mechanical properties in crystalline materials.

Simple and easy-to-characterize molecular crystals of a related set of compounds with limited variables provide a good starting point for a regressive analysis to evaluate various contributing elements to the observed mechanical traits. ${ }^{19}$ To date, elasticity and plasticity in crystals are perceived in terms of molecular packing and the relative distribution of interaction types having varying strengths within the crystal. The isotropic distribution of robust electrostatic interactions and close-packed molecular arrangments add brittleness to the crystals, whereas weak dispersive interactions favor elasticity. ${ }^{20,21}$ Layered molecular arrangement with an orthogonal distribution of strong and weak interactions ensue plasticity in the system. ${ }^{22,23}$ Though the aforesaid factors are critical structural aspects that cause distinct mechanical characteristics of molecular crystals, various other factors-solvent of crystallization, ${ }^{24-26}$ globularity of the molecules, ${ }^{16,27}$ and aspect ratio of the crystals ${ }^{3}$-also could contribute to their mechanical properties. Also, one could employ crystal engineering strategies to tune the mechanical properties in molecular crystals. ${ }^{28-30}$

Interestingly the elasticity and plasticity are orthogonal mechanical properties, having contrasting structural requirements and contributing factors. Their co-existence hence appears contradictory and is exceptional. ${ }^{31,32}$ The genesis of the present study is rooted in an earlier report on the co-existence of elasticity and plasticity in the crystals of bromobenzil, ${ }^{31}$ wherein crystals notably undergo reversible to irreversible bending under the varying extent of applied stress. Beyond the elastic domain, the deformation becomes irreversible, rather than breaking. In the present work, we explore this unique property and the scope of the generality of the 
observation. We studied a series of benzil compounds (Scheme 1) and a related set of oxalate compounds having halogen substituents in the $p$-carbon atom; most of the compounds have similar global packing mode-layered structure. In the course of the study, we identified a novel crystal form of the F-derivative (BZF)and the dimorphs exhibit distinct mechanical response under applied stress. The benzil crystals show intense emission at the crystal ends, in both straight and bended state, compared to the middle areas, hinting their optical wave guiding properties. Such molecular crystals with exceptional flexibility and emitting characteristics are rare. This work aims at expounding the microscopic and macroscopic factors that contribute to the rare and exceptional co-existence of mutually orthogonal mechanical traits.
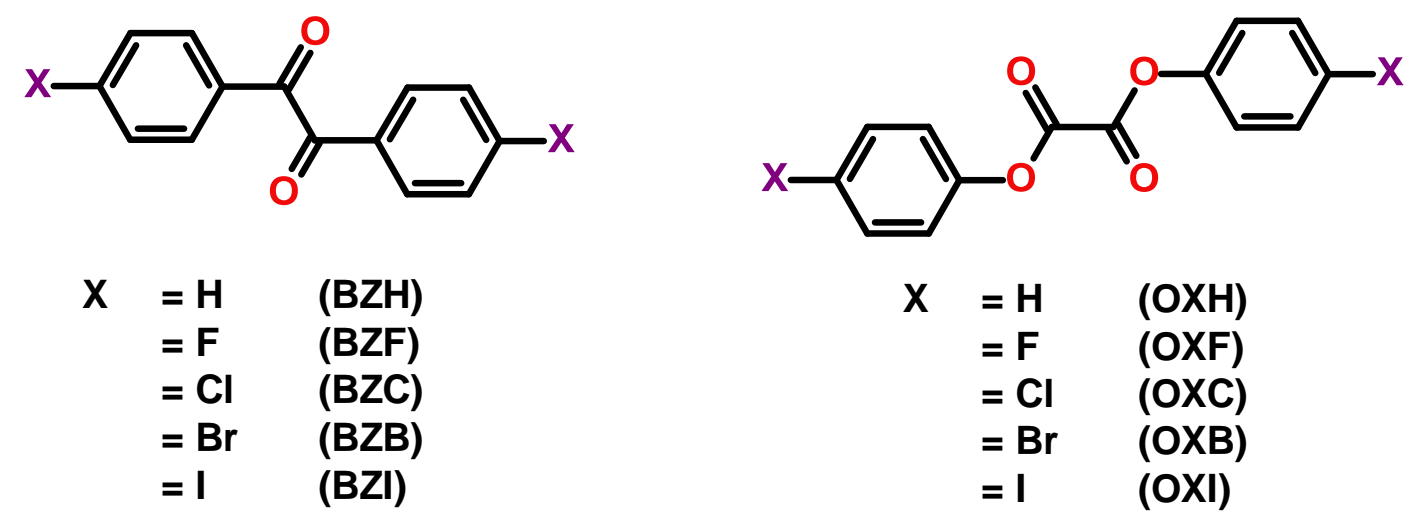

Scheme 1 The molecular structures of the benzil and oxalate compounds studied in the present contribution.

\section{Results and Discussion}

To verify the structural relations and the mechanical properties, we investigated the crystal structure of the benzil compounds with halogen-substituted in the p-position of the phenyl rings, analogous to the Br-derivative reported in a previous publication. Slow evaporation of 1:1 dichloromethane: methanol/ethanol solutions yielded the long tape-like crystals of BZC and BZB. The methanol solution of the fluoro-derivative yielded pale yellow block-like crystals of BZF_O, while a 1:1 dichloromethane: methanol mixture yielded long tape-like crystals of BZF.

\section{Structure and mechanical properties of benzil compounds}

Under applied mechanical stress, thin tape-like crystals of the benzil compound BZB exhibit good elasticity. The crystals of BZB could bend elastically into a loop, by three-point bending methods. 
(a)

(b)

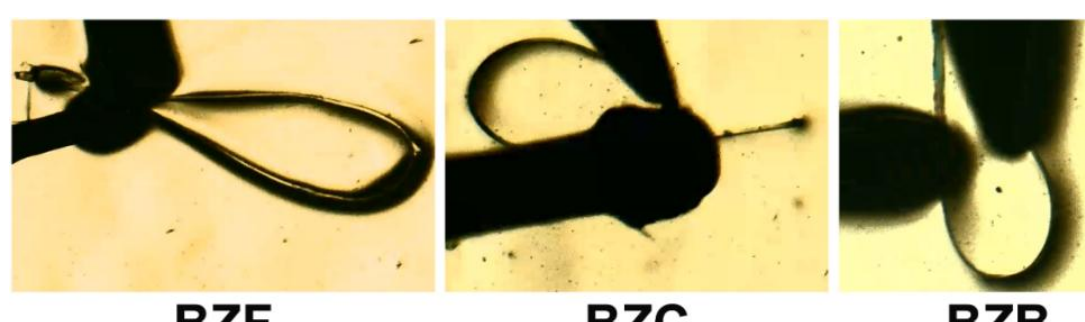
BZF
BZC
BZB
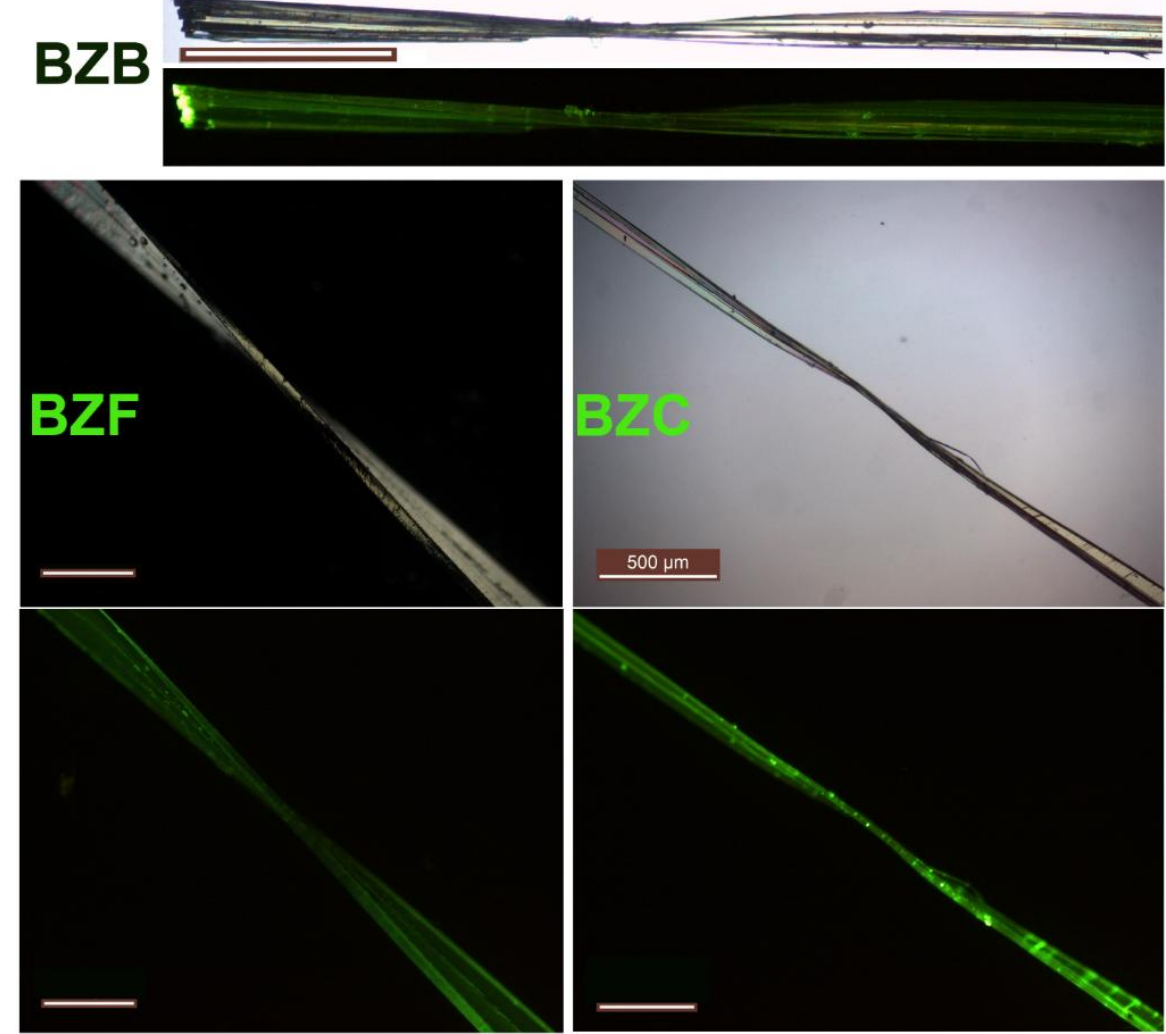

(c)
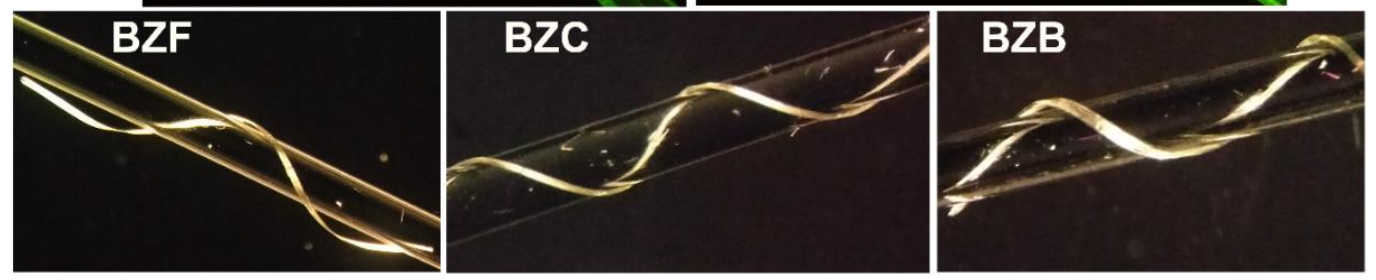

Fig. 1 The crystals of BZF, BZC, and BZB under different deformation regimes. (a) elastic; (b) twisted and (c) coiled around a capillary tube.

Small deformation stress, leading to a bending strain $(\varepsilon)$ of $\sim 2.45 \%$ on a crystal with thickness $\sim 65 \mu \mathrm{m}$, resulted in elastic deformation (Table S3). The bending in BZB crystal is associated with the $\{020\}$ face, while it cracked when other faces were deformed. The bending behavior could be repeated without fatigue. Excessive, $\varepsilon$ beyond $\sim 2.45 \%$, bending of the crystal does not lead to its breakage but macroscopic plastic deformation (Fig. S1). Akin to the recent observation of the plastic bending with some extent of elastic recovery, ${ }^{33}$ the crystals of BZB show partial elastic recovery even after the crystals are plastically deformed $(\varepsilon=1.26 \%)$. The elastic to plastic bending characteristics and the elastoplastic trait of the BZB can be 
intuitively observed in the Movie S1 of ESI. Thus, the crystal is an archetype system wherein the two distinct mechanical traits with orthogonal structural origin co-exist. A similar observation was made in the case of BZC, wherein both the elastic and plastic properties coexist. The elasticity in BZC is associated with $\{002\}$ crystal face, and akin to BZB, excess mechanical stress ( $>\varepsilon=2.39 \%$ ) leads to plastic deformation (Fig. S2). The bending-relaxation process in benzil crystals is repeatable, highlighting its excellent elasticity. Of note, the crystals of both BZB and BZC can be looped but, beyond the elastic strain it undergoes plastic deformation (Movie S2). The crystals can be readily deformed into contorted shapes, twisted, or even coiled around a capillary tube in the plastic regime (Fig. 1).

(a)
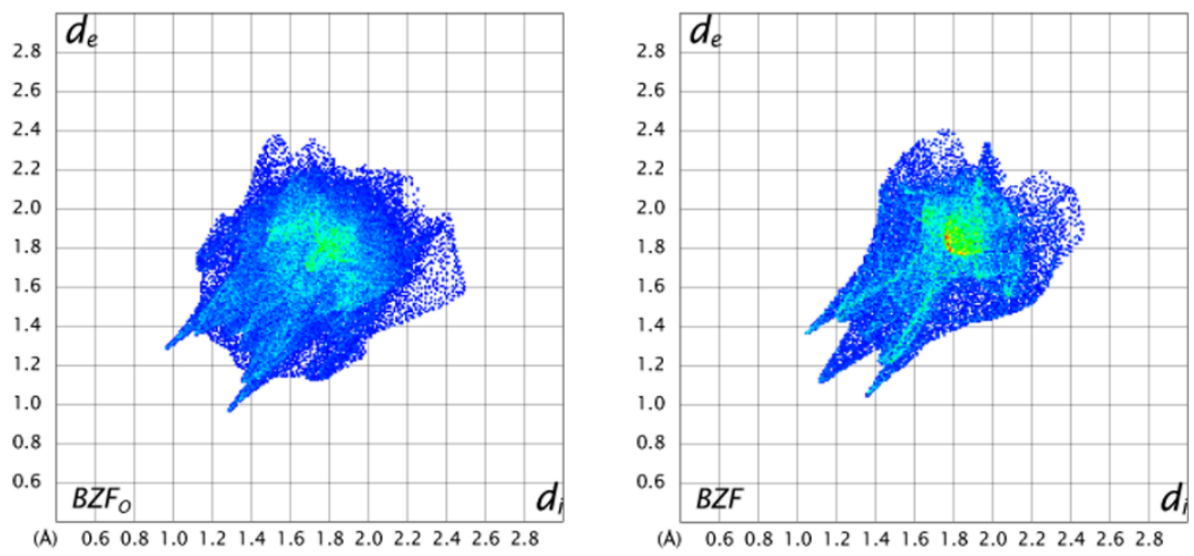

(b)
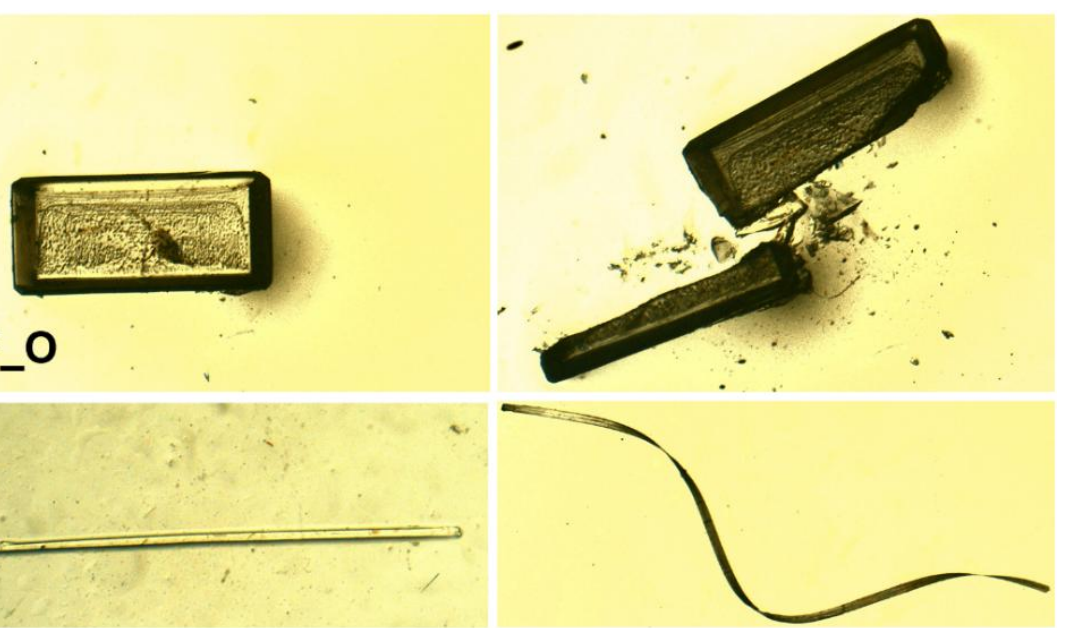

Fig. 2 The dimorphs of the benzil F-derivative (BZF_O and BZF): (a) HS fingerprint plot highlights the difference in the interactions; (b) distinct mechanical characteristics of the dimorphs.

The polymorphs of the F-derivative (BZF_O and BZF) exhibit distinct mechanical properties. While block-like crystals of BZF_O are brittle, the long tape-like crystals of BZF could be mechanically deformed based on the extent of the applied stress. Besides getting elastically deformed to a loop ( $\varepsilon=2.36 \%$ ) (Fig. S3), the crystals of BZF can also be plastically twisted (twist angle of $\sim 180^{\circ}$ ), wrapped (out of plane) (Fig. S10, S17) or coiled around a 
capillary (Fig. 1c). Compared to BZB and BZC, the crystals of BZF are more viable for bending and twisting. The crystal face associated with the bending in BZF is $\{020\}$. The block-like crystals of BZF_O, in contrast, have a close-packed molecular arrangement and an isotropic distribution of the interactions, making it devoid of any energetically viable slip plane, hence brittle (Fig. 2b).

(a)
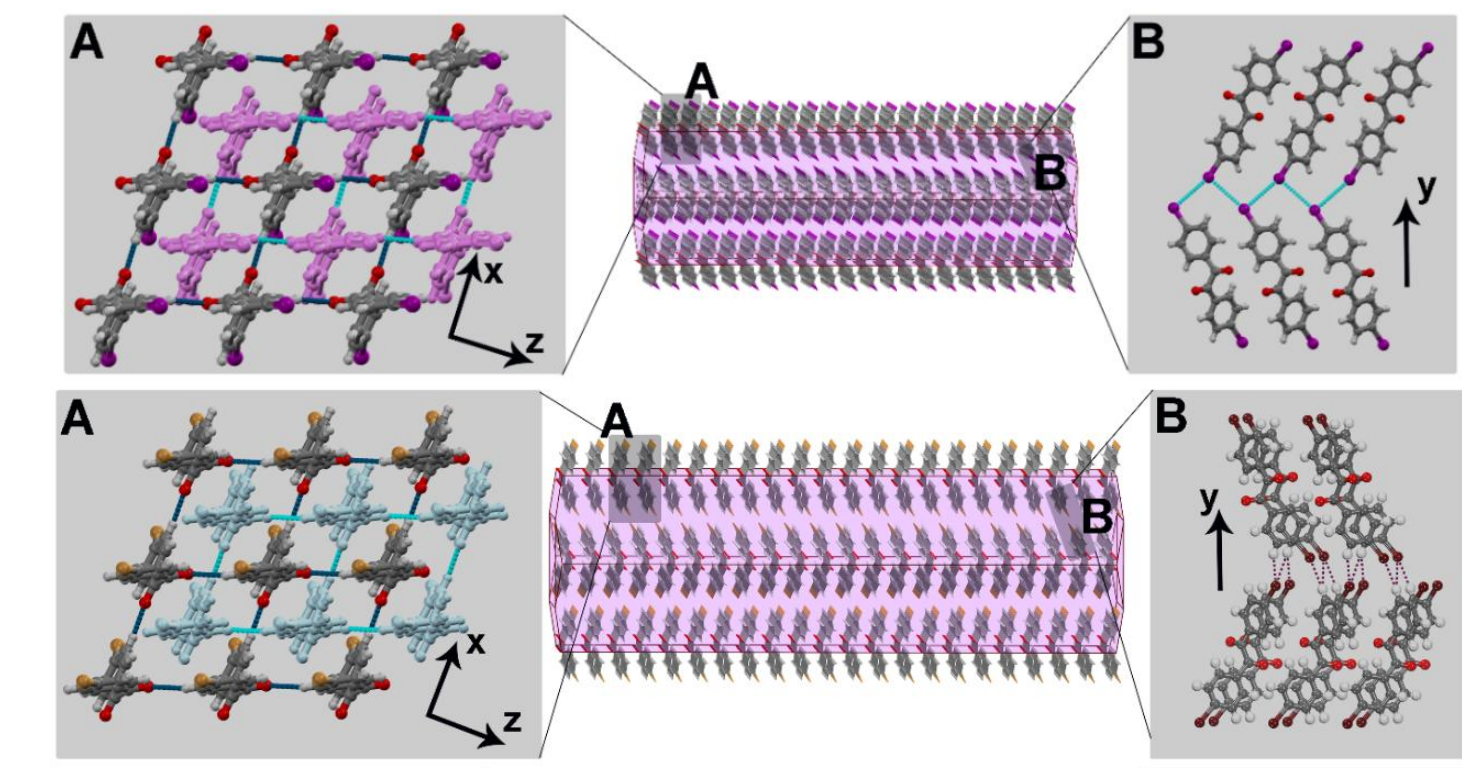

(b)

(c)
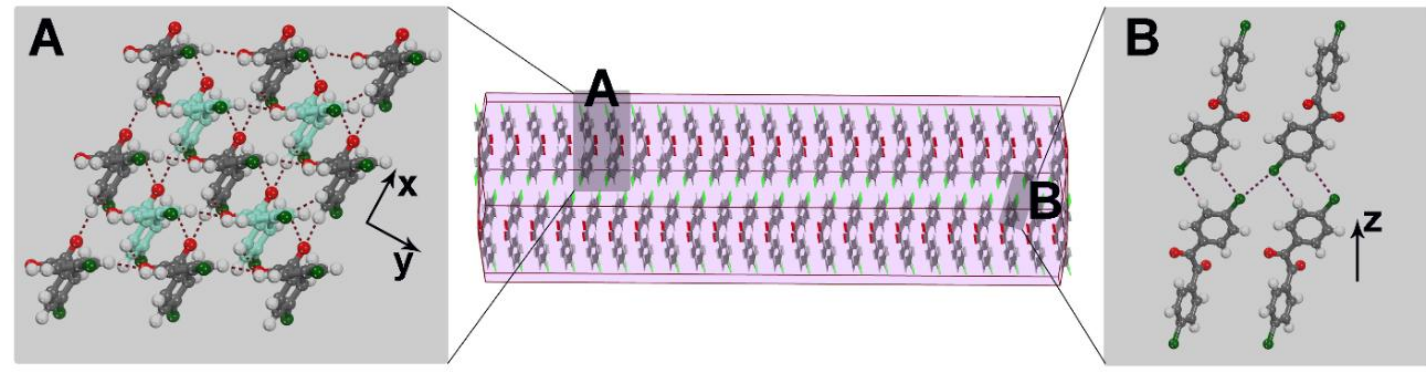

(d)
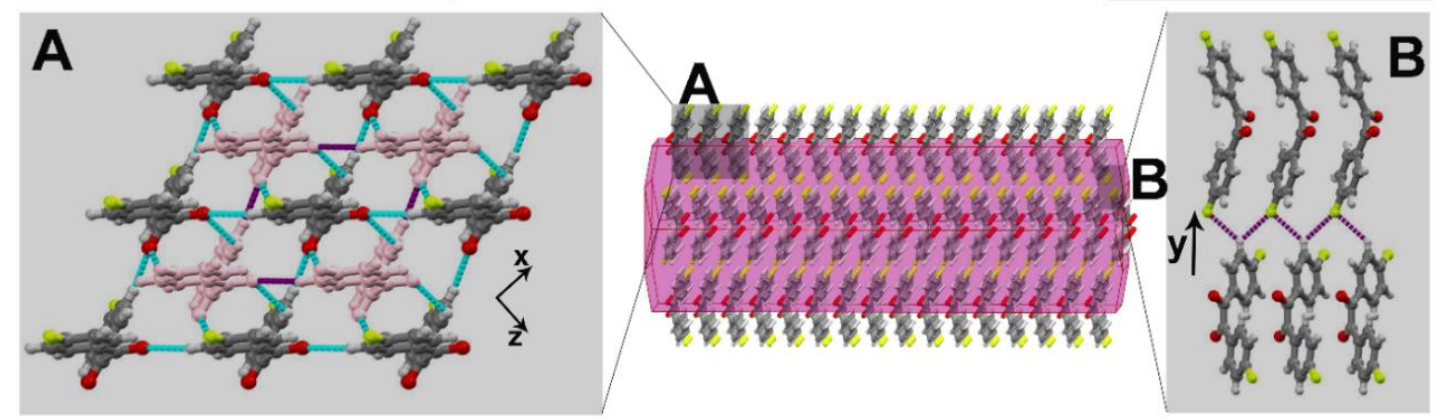

Fig. 3 The computed crystal morphology and molecular packing. (a) BZI; (b) BZB; (c) BZC and (d) BZF. Note the similar stacking arrangement though the interlayer region shows distinct interaction types.

To gain insights into the molecular-level mechanism of the intricate co-existence of elasticity and plasticity in the crystals, we analyzed the crystal structure of the benzil compounds in terms of crystal packing and interactions. In the Br-derivative (BZB), the measured torsion about the diketo $(\mathrm{O}=\mathrm{C}-\mathrm{C}=\mathrm{O})$ moiety is $116^{\circ}$, whereas the planes containing 
the aromatic rings describe an angle of $57^{\circ}$ (Table S4). The molecules adopt a stacked-layered arrangement. Within a layer, the molecules make a grid-like arrangement in the $a c$-plane, and along [010], adjacent grids interweave through a network of $\mathrm{C}-\mathrm{H} \cdots \mathrm{O}(2.49 \AA)$ and $\pi \cdots \pi(3.54$ $\AA$ ) interactions (Fig. 3). The interlayer region $\beta$ with Type-II $\operatorname{Br} \cdots \operatorname{Br}\left(\theta_{1}=162\right.$ and $\left.\theta_{2}=95^{\circ}\right)$ and $\mathrm{C}-\mathrm{H} \cdots \mathrm{Br}(3.19 \AA)$ interactions form potential slip planes parallel to $\{010\}$. The I-derivative (BZI) is isostructural to BZB and has similar interaction types and packing arrangements. In the crystal structure, relatively more robust interactions form 2D layer; the Type-II I $\cdots I$ I $\left(\theta_{1}=164\right.$ and $\left.\theta_{2}=100^{\circ}\right)$ and $\mathrm{C}-\mathrm{H} \cdots \mathrm{I}(3.34 \AA)$ interactions occupy the interlayer region and describe the slip planes. In the chloro-compound (BZC), the overall packing adopted by the molecule is comparable to BZB and can be described as a stacked-layered type. A unique molecule of BZC, the diketo moiety makes bifurcated $\mathrm{C}-\mathrm{H} \cdots \mathrm{O}(2.47$ and $2.70 \AA$ ) hydrogen bonds with three molecules (two from the same grid and one from the adjacent one). Further to $\mathrm{C}-\mathrm{H} \cdots \mathrm{O}$ hydrogen bonds, $\pi \cdots \pi$ (average $3.45 \AA$ ) interactions also significantly contribute to layer formation. The (001) defines the interlayer region dominated by Type I Cl $\cdots \mathrm{Cl}\left(\theta_{1}=\theta_{2}=164^{\circ}\right)$ and $\mathrm{C}-\mathrm{H} \cdots \mathrm{Cl}(3.06 \AA$ ) interactions, which form the slip plane.

The known crystal form of the F-derivative (BZF_O) has a structure distinct from the rest in the series. The acicular crystals of the novel polymorph BZF, as the diffraction studies establish, is isostructural to the rest of the halo-derivatives. The compound crystallizes in an orthorhombic $P 2{ }_{1} 2{ }_{1} 2$ space group with an overall packing described as stacked-layered type. Within a layer, molecules make square grid-like structures along the (101) plane, and the adjacent grid structures interweave through $\mathrm{C}-\mathrm{H} \cdots \mathrm{O}$ hydrogen bonds along [001]. Each diketo moiety in the compound form bifurcated $\mathrm{C}-\mathrm{H} \cdots \mathrm{O}(2.54$ and $2.78 \AA$ ) hydrogen bonds. Further to $\mathrm{C}-\mathrm{H} \cdots \mathrm{O}$ hydrogen bonds, $\pi \cdots \pi$ (average $3.60 \AA$ ) interactions also significantly contribute to layer formation. In the [010], the layers are connected through $\mathrm{C}-\mathrm{H} \cdots \mathrm{F}(2.73$ and $2.75 \AA$ ) hydrogen bonds, wherein each F-atom forms bifurcated interactions. The twistability of BZF crystals are rooted in the two directional molecular orientation and multifacitated bending, which enable the crystals to bend along two different directions.

Thus, in the halo-derivatives of benzils, ${ }^{34}$ the overall structural topology remains comparable, though there is a notable variation in the interactions types when analyzed in greater detail. The $\mathrm{C}=\mathrm{O}$ of the diketo moiety makes either bifurcated $\mathrm{C}-\mathrm{H} \cdots \mathrm{O}$ hydrogen bonds (as in BZC and BZF) or single $\mathrm{C}-\mathrm{H} \cdots \mathrm{O}$ interaction (as in BZB and BZI). Besides the $\mathrm{C}-\mathrm{H} \cdots \mathrm{O}$ hydrogen bonds, all the compounds have $\pi \cdots \pi$ interactions as a major stabilizing force, though their average distance increase as one moves from BZB to BZF in the series. The interlayer region that constitutes the slip planes has distinct interactions. BZB has Type-II $\mathrm{Br} \cdots \mathrm{Br}$ together 
with $\mathrm{C}-\mathrm{H} \cdots \mathrm{Br}$ interactions, while Type $\mathrm{I} \mathrm{Cl} \cdots \mathrm{Cl}$ and $\mathrm{C}-\mathrm{H} \cdots \mathrm{Cl}$ interactions occupy the interlayer region in BZC. In BZF, C-H $\cdots \mathrm{F}$ interactions organize the interlayer region. Thus, the benzil compounds show a systematic transition in the interaction type and energetics in the interlayer region. The twisted conformation of the central diketo moiety enables the molecules to make interwoven grid-like networks in the intralayer region defined by a combination of $\pi \cdots \pi$ and $\mathrm{C}-\mathrm{H} \cdots \mathrm{O}$ interactions. In the absence of classic electrostatic interactions, the dispersive interactions provide adequate flexibility to undergo elongation and compression, thereby a structural buffer to accommodate the excess strain generated within the crystal. Such structural flexibility essentially promotes reversible deformation; in contrast, the stacked-layered arrangement and the interlayer region sustained through weaker interactions provide the impetus to deform irreversibly. The weak $\mathrm{X} \cdots \mathrm{X}$ and $\mathrm{C}-\mathrm{H} \cdots \mathrm{X}$ interactions afford initial resistance to a long-range slippage of layers along the slip planes, facilitating initial elasticity followed by plasticity at higher strains.

To further explore the nuance and the intricate relation of structure and mechanical properties, we correlated them with a series of oxalate compounds with halogen-substituted at the $p$-position. In the series, we wanted to explore the possible anisotropy brought about by the $\pi$-hole interactions by the central oxalate moiety. The oxalate functionality remains planar, though it is near orthogonal to the coplanar aromatic rings. ${ }^{35}$ The coplanar aromatic rings are optimal to form $\pi \cdots \pi$ interactions. Thus with weaker interactions in the interlayer region, the system develops enough anisotropy to alter the delicate balance between elasticity and plasticity. The torsional shifts in the compounds are provided in Table S5.

\section{Structure and mechanical properties of oxalate compounds}

Oxalate crystals also exhibit the property of the co-existence of elastic and plastic deformation, though the elastic domain is considerably lesser vis-à-vis the benzil compounds. The block-like crystals of OXH and needles of OXF are brittle and crack under applied mechanical stress. However, the crystals of OXC and OXB can be elastically bent to semicircles; but, they fail to curl into a loop (Movie 5 and 6). The crystals do not crack beyond the elastic strain limit but deform plastically (Fig. S4 and S5). The plastic deformation is dominant in the oxalate series with notably less elastoplastic characteristics, as compared to the benzil compounds. Further, an increase in the thickness of crystals diminishes the elastic region making the crystals undergo plastic deformation directly. Though the crystals of oxalates deform plastically under applied stress, they are not viable for twisting. The OXI crystals do not bend; instead, they can be sheared along the $\{002\}$ plane, which is defined by 
I...I interactions (Fig. 4). The observed mechanical properties in the oxalate can be correlated to microscopic structural factors such as crystal packing and interaction types.

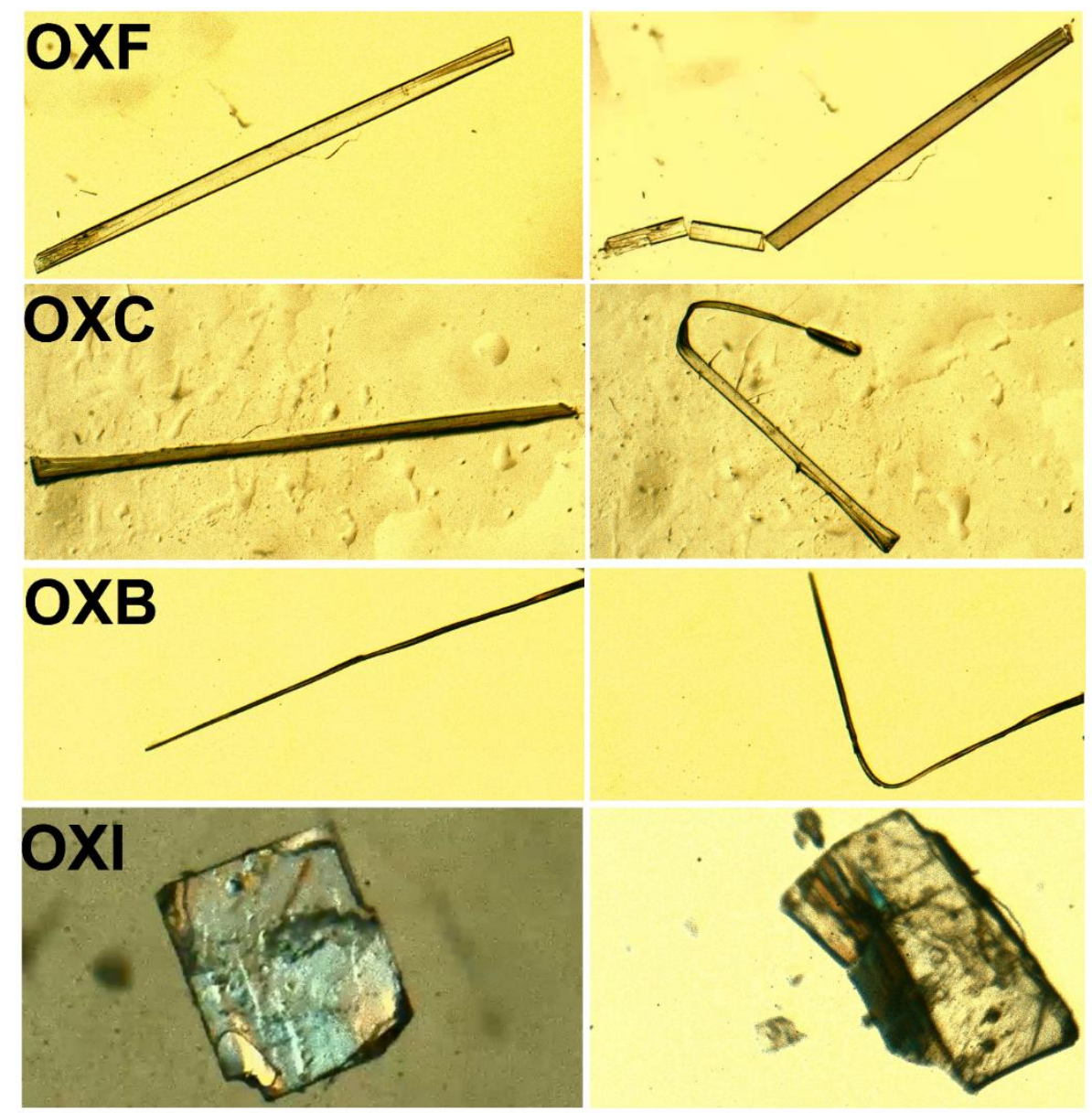

Fig. 4 The mechanical properties in the oxalate compounds. The OXF crystals are brittle, while OXC and OXB show plastic bending. The block-like crystals of OXI undergo shearing under applied stress.

$\mathrm{OXB}$ and $\mathrm{OXC}$ have similar structures with the formation of a stacked structure and the morphology as long thin tape-like crystals with $\{100\}$ as the prominent face (Fig. 5). Along the [010], OXB molecules make linear chains through $\pi \cdots \pi(3.46 \AA)$ and $\mathrm{C}=\mathrm{O} \cdots \mathrm{C}(3.20 \AA)$ interactions. The structure is defined by the distribution of relatively more robust interactions along the $b c$-plane; Type- $\mathrm{I} \operatorname{Br} \cdots \operatorname{Br}\left(\theta_{1}=\theta_{2}=153^{\circ}\right)$ and $\mathrm{C}-\mathrm{H} \cdots \mathrm{Br}(3.34 \AA$ and $3.48 \AA)$ define the interlayer region, the (100) plane, which acts as the slip plane. OXC has a layered structure wherein adjacent chains are connected along [001] through bifurcated $\mathrm{C}-\mathrm{H} \cdots \mathrm{O}(2.65$ and $2.71 \AA$ ) and $\mathrm{C}-\mathrm{H} \cdots \mathrm{Cl}(2.93 \AA$ ) interactions. The (100), which corresponds to the slip plane, contains the Type- $\mathrm{I} \mathrm{Cl} \cdots \mathrm{Cl}\left(\theta_{1}=\theta_{2}=162^{\circ}\right)$ contacts and weak dispersive $\mathrm{C}-\mathrm{H} \cdots \mathrm{Cl}(3.11 \AA)$ and $\mathrm{C}-\mathrm{H} \cdots \pi(3.10 \AA)$ interactions. In OXF, molecules form linear chains through centric $R_{2}^{2}(12) \mathrm{C}-$ $\mathrm{H} \cdots \mathrm{O}(2.66 \AA)$ hydrogen bonds along [100] and further stacked through $\pi \cdots \pi(3.54 \AA)$ interactions along [001]. A network of weak dispersive interactions $(\mathrm{C}-\mathrm{H} \cdots \mathrm{O}(2.66 \AA), \mathrm{F} \cdots \mathrm{C}$ 
(3.05 $\AA$ ) and $\mathrm{C}-\mathrm{H} \cdots \pi(3.03 \AA)$ along $[011]$ lead to an intercalated structure, wherein the stacking of linear chains leads to the formation of a close-packed structure.

In the stacked-layered structure of OXI, a layer consists of zig-zag chains (along [010]) of the molecules connected through $\pi \cdots \pi$ (3.37 and $3.25 \AA$ ), $\mathrm{C}=\mathrm{O} \cdots \mathrm{C}(2.95$ and $2.97 \AA$ ), and $\mathrm{C}-\mathrm{H} \cdots \mathrm{O}(2.79$ and $2.86 \AA)$ interactions. These zig-zag chains further connect through $\pi \cdots \pi$ (3.47 and 3.41 $\AA$ ), $\mathrm{C}=\mathrm{O} \cdots \mathrm{C}(3.07$ and 3.17 $\AA$ ), and $\mathrm{C}-\mathrm{H} \cdots \mathrm{O}(2.80 \AA$ ) interactions along [100] to form a two-dimensional layer structure. The interlayer region is stabilized through a network of Type-II I $\cdots \mathrm{I}\left(\mathrm{C}-\mathrm{I} \cdots \mathrm{I}\right.$ angle of 165 and $\left.114^{\circ}\right)$ interactions. OXH, unlike the rest of the series, has the phenyl substitution in syn-conformation (Table S5) and the aromatic rings twisted with respect to each other $\left(22.56^{\circ}\right)$. In the [100], molecules make centric dimers through $\mathrm{C}-\mathrm{H} \cdots \mathrm{O}$ $(2.574,2.586 \AA)$ hydrogen bonds and are further connected through $\pi \cdots \pi(3.058 \AA$ ) interactions to form a linear chain. The linear molecular chains thus formed are held through a network of $\mathrm{C}-\mathrm{H} \cdots \pi$ and $\pi \cdots \pi$ interactions along $[010]$ and $[001]$ to form a three-dimensional structure. The brick-wall crystal packing and the criss-cross arrangement of interactions make OXH brittle.

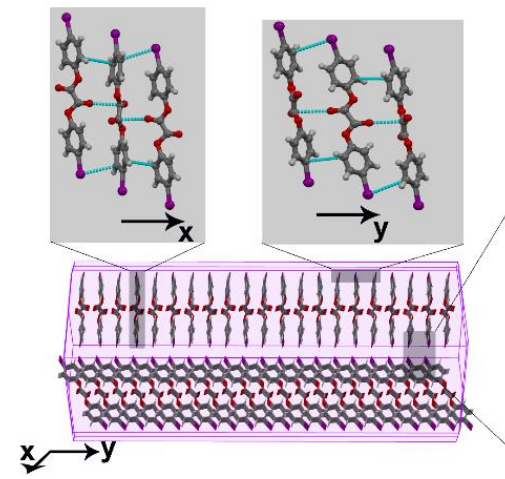

(a)

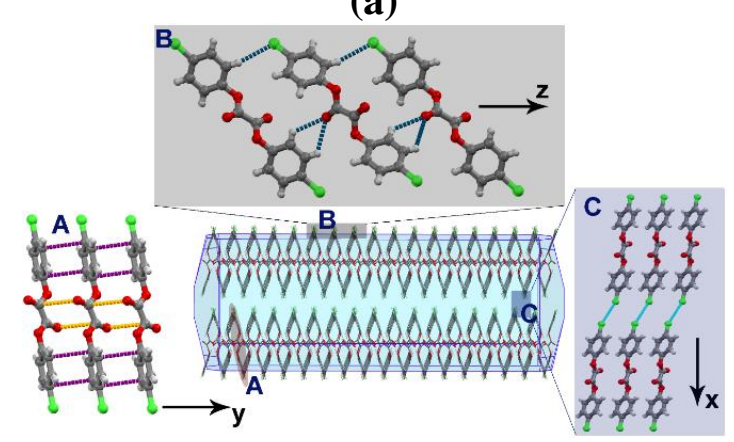

(c)

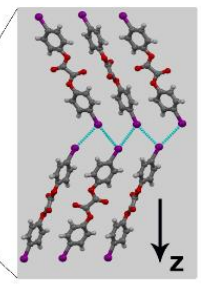

z

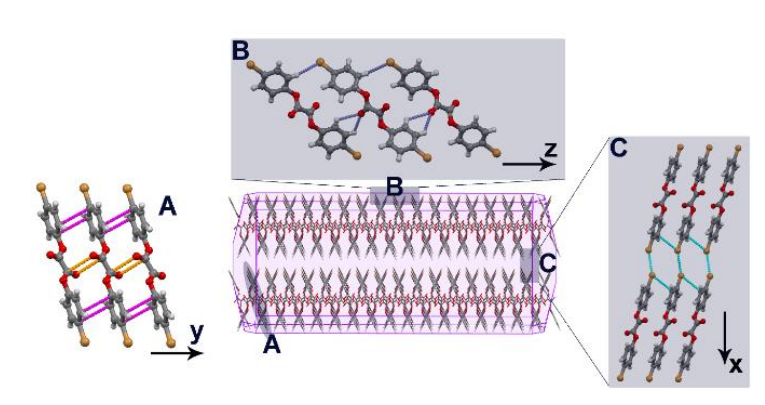

(b)

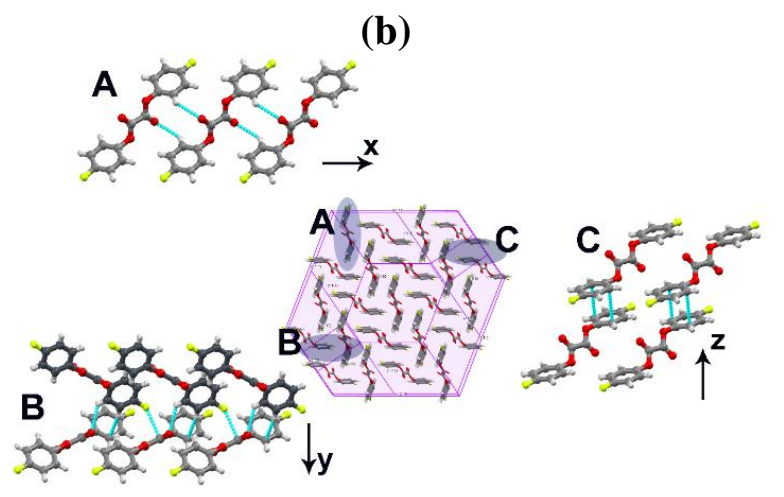

(d)

Fig. 5 The crystal morphology and molecular packing in (a) OXI; (b) OXB; (c) OXC and (d) OXF.

\section{Analysizing the structural elements that contribute to the mechanical properties}

As manifested by twistable crystals, multiple bendable faces involve more crystal isotropy - a requirement for elasticity; simultaneously, it opposes the prerequisite of plastic 
bending - the interaction anisotropy. Thus, two contrasting mechanical characteristics co-exist in the crystals of benzil compounds. The microscopic structural and macroscopic morphological factors were explored to gain insights into this unique co-existence. The structures are devoid of classical electrostatic interactions in the set of crystals studied in the present work. Hence, regulating the distribution of dispersive interactions is critical in tuning the delicate balance of interactions and further understanding the anisotropy in determining the macroscopic mechanical properties. Towards this, we explored the role of the linker moiety (Z) in the $\mathrm{X}-\mathrm{Ar}-\mathrm{Z}-\mathrm{Ar}-\mathrm{X}$ compound types in attaining a non-planar conformation, modifying the interaction types, and inducing their influence in the structural anisotropy. The mechanical properties of Schiff base $(-\mathrm{CH}=\mathrm{N}-)$ amide $(-\mathrm{NH}-\mathrm{CO}-)$ and ester $(-\mathrm{O}-\mathrm{CO}-)$ moieties are known to induce distinct mechanical properties but, benzil and oxalate entities are unique.

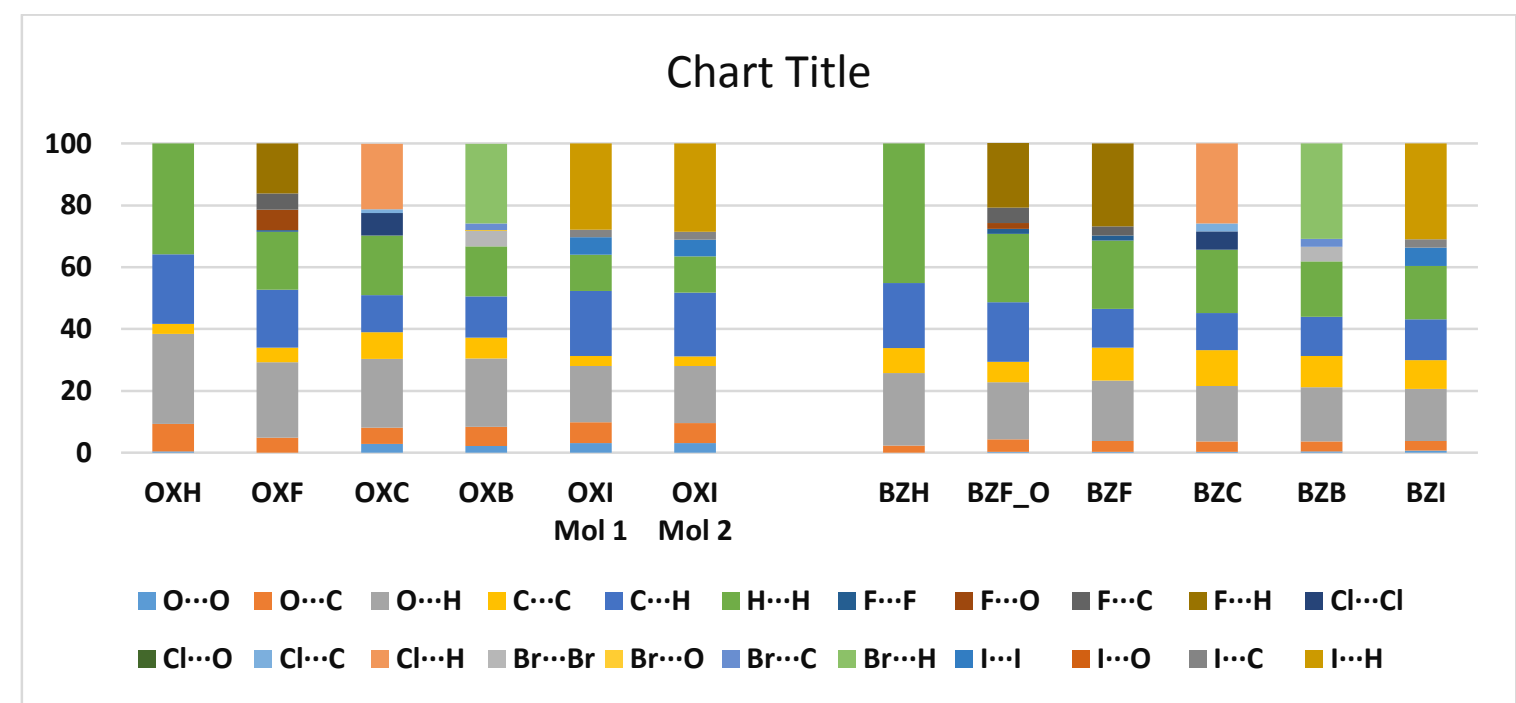

Fig. 6 The relative contribution of interactions, as derived from the Hirschfeld analysis.

Hirschfeld Surface (HS) analysis: The HS analysis of the relative contribution of interactions helps to derive possible correlations between the interaction types and the observed molecular characteristics. ${ }^{36}$ In a comparative analysis, the respective central moieties in the oxalate and benzil series are critical in the structure formation, hence their macroscopic mechanical properties. The rigid and coplanar central oxalate moiety enables an enhanced contribution of $\mathrm{C}=\mathrm{O} \cdots \mathrm{C}$ interactions, compared to the twisted diketo moiety in benzil compounds. Thus, the $\mathrm{O} \cdots \mathrm{C}$ contribution is $>50 \%$ higher in a unique oxalate compound in comparison to its analogous benzil derivative. 
BZH

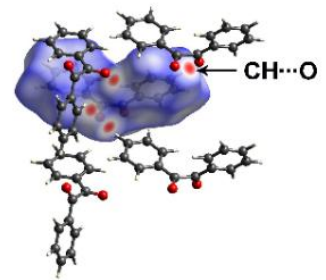

BZF

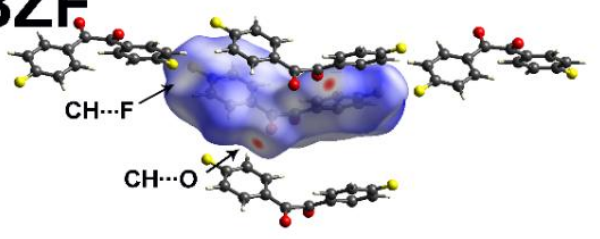

BZC
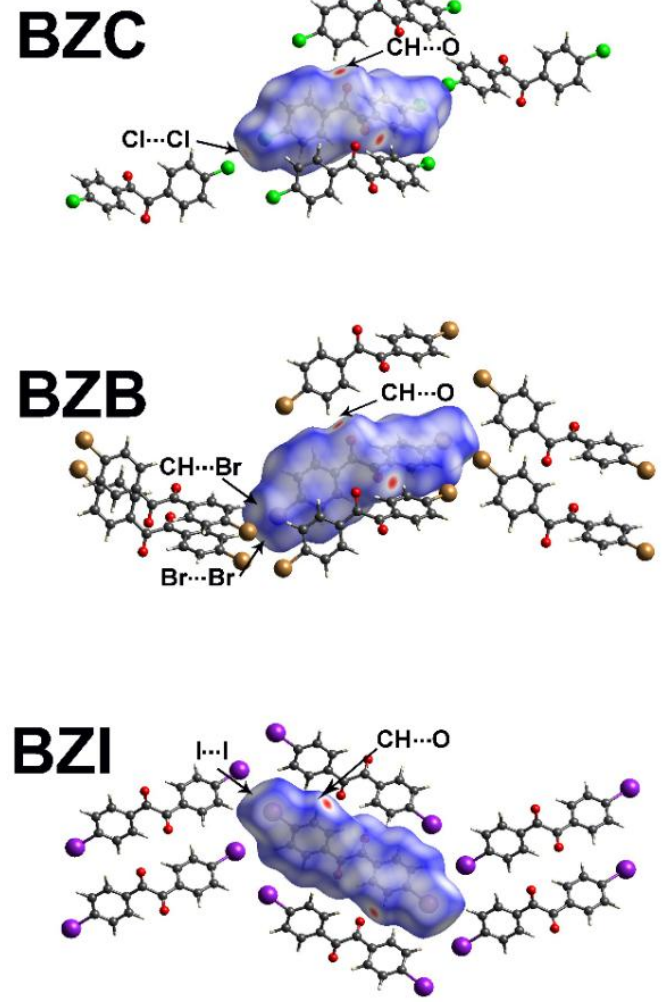

$\mathrm{OXH}$

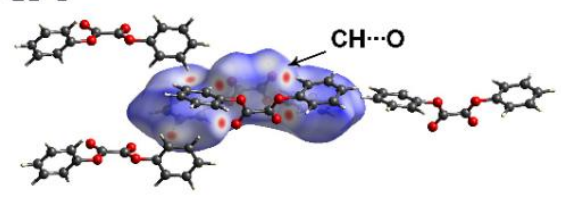

\section{OXF}
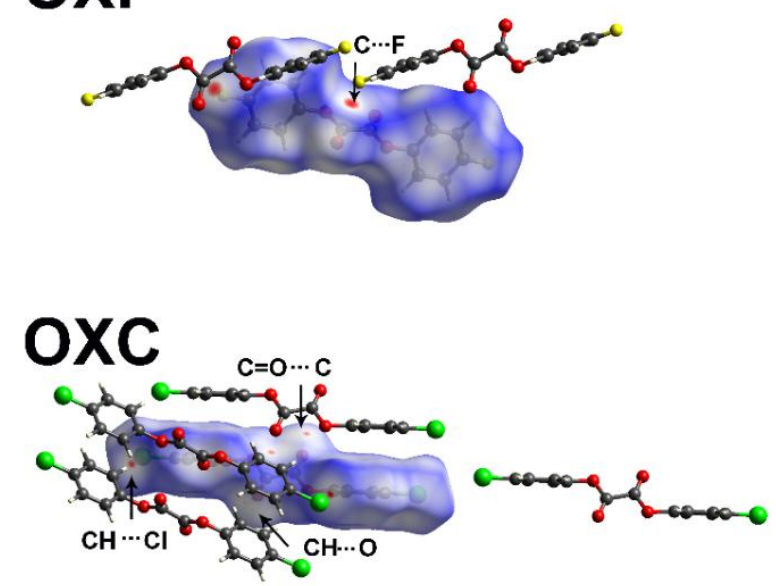

\section{OXB}
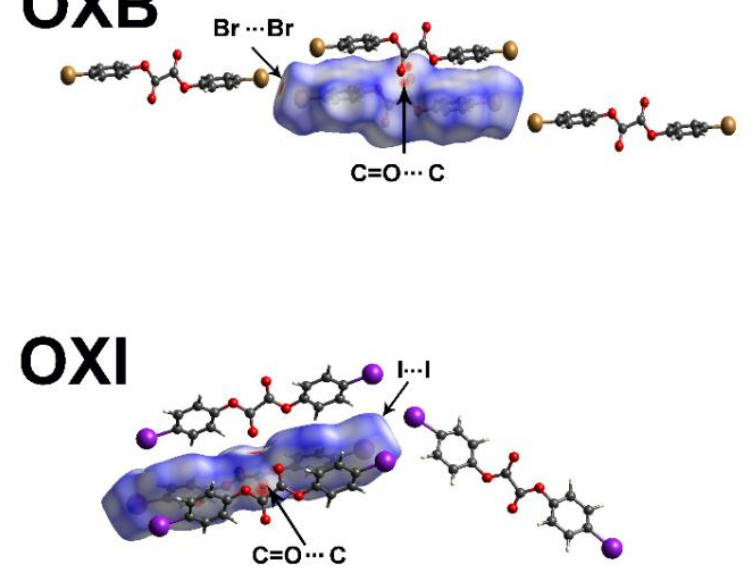

Fig. 7 Hirshfeld surface with $d_{\text {norm }}$ mapped over it using universal red, white and blue color code that indicates strong, medium, and weak interactions, respectively. The prominent interaction types are highlighted.

Also, a higher number of O-atoms available in the oxalates vis-à-vis benzil compounds leads to an apparent increase in the contribution of $\mathrm{O} \cdots \mathrm{H}(\mathrm{C}-\mathrm{H} \cdots \mathrm{O})$ interactions. The interaction types established by the halogen atoms secure the interlayer region. The fluorine in BZF makes a network of $\mathrm{C}-\mathrm{H} \cdots \mathrm{F}$ interactions in the interlayer region with a prominent contribution in the structure formation (26.9\%) as against $16.2 \%$ in OXF-a notable $66 \%$ increase. The $\mathrm{C} \cdots \mathrm{F}$ contacts, however, are comparable in the analogous compounds. 
Considering the three types of interactions $(\mathrm{Cl} \cdots \mathrm{Cl}, \mathrm{Cl} \cdots \pi$ and $\mathrm{C}-\mathrm{H} \cdots \mathrm{Cl})$ involving the $\mathrm{Cl}$ atom, the $\mathrm{Cl} \cdots \mathrm{Cl}$ interaction stand out in $\mathrm{OXC}$ as against $\mathrm{C}-\mathrm{H} \cdots \mathrm{Cl}$ in $\mathrm{BZC}$. The contribution of $\mathrm{Br} \cdots \mathrm{Br}$ in both $\mathrm{OXB}$ and $\mathrm{BZB}$ is comparable; the benzil compound in addition has significant contribution of $\mathrm{C}-\mathrm{H} \cdots \mathrm{Br}$ interaction (20\%). A similar trend subsists in the Iderivatives (Fig. 6). The enhanced contribution of the $\mathrm{X} \cdots \mathrm{H}$ interactions in the benzil compounds can be attributed to their twisted conformation and these interactions in the interlayer region help to ease the structural anisotropy.

The HS, when mapped along with unique interactions, provides information on the nature and strength of interactions, which is critical in determining the mechanical properties (Fig. S26 and S27). The HS of OXH has more points of contact with the neighboring molecules than BZH. The electron-rich F-atom in OXF make $\pi$-hole $\mathrm{C}-\mathrm{F} \cdots \mathrm{C}=\mathrm{O}(3.05 \AA)$ interactions with the $s p^{2} \mathrm{C}$ - atom of the carbonyl group. In the rest of the halo-derivatives of oxalates, the carbonyl group makes carbonyl-carbonyl $(\mathrm{CO} \cdots \mathrm{CO})$ tetral bonds. The $\mathrm{OXC}$ and $\mathrm{OXB}$ make $\mathrm{CO} \cdots \mathrm{CO}$ contacts, and depending on the relative orientation of the interacting carbonyl groups, they are classified as Motif-II, ${ }^{37}$ with $\mathrm{C} \cdots \mathrm{O}=\mathrm{C}$ distance $\left(d_{\mathrm{CO}}\right)$ of 3.24 and $3.10 \AA$ and having the $\mathrm{O} \cdots \mathrm{C}=\mathrm{O}$ angle of 84 and $83^{\circ}$, respectively. The measured angle of the $\mathrm{OC}=\mathrm{O}$ is lower than the average value of $92.6^{\circ} 38,39$ and that of the Burgi-Dunitz (BD) angle $\left(107^{\circ}\right){ }^{38,39}$ In OXI, the symmetry-independent molecules make CO $\cdots \mathrm{CO}$ interaction of Motif-I type but with distinct parameters; the $d_{\mathrm{CO}}$ is 2.95 and $3.20 \AA$, and angle of 110 and $103^{\circ}$.

Energy framework $(E F)$ analysis: The information on the interaction types and their relative distribution in the crystal structure in terms of EF analysis, together with the molecular arrangement, offers to draw structure-mechanical property correlations. ${ }^{40} \mathrm{In} \mathrm{BZH}$, the total energy representation of the EF is effectively two-dimensional; nevertheless, the close-packed crystal structure and hence the lack of an energetically viable slip system makes it brittle. The brittle BZF_O crystals have a close-packed structure with criss-crossed EF, while the deformable novel polymorph BZF has a layered structure with a 2D network (Fig. S28). Thus, distinct crystal structures and different distribution of interactions lead to explicit variations in the mechanical response in the polymorphs. In $\mathrm{BZC}$, the $\mathrm{Cl} \cdots \mathrm{Cl}$ interaction being Type-I $\left(\theta_{1}=\right.$ $\theta_{2}=164^{\circ}$ ) and weak, the calculated EF is per se two dimensional and enables the layers to slip with respect to each other. The EF analysis highlights the isostructurality of BZB and BZI in terms of overall crystal packing and the interaction types that sustain the interlayer region. In $\mathrm{BZB}$, the Type-II $\mathrm{Br} \cdots \mathrm{Br}$ interaction is $\left(\theta_{1}=163^{\circ}\right.$ and $\left.\theta_{2}=94^{\circ}\right)$ is longer than the sum of the van der Waals radii by $0.15 \AA$. Based on the EF analysis (Fig. S28), the Type-II interaction is more electrostatic than dispersive, with an energy component of $\sim-6 \mathrm{~kJ} / \mathrm{mol}$. The value agrees 
with the reported value of $7 \mathrm{~kJ} / \mathrm{mol}^{41}$ In the case of BZI, the Type-II $\left(\theta_{1}=165^{\circ}\right.$ and $\left.\theta_{2}=99^{\circ}\right)$ $\mathrm{I} \cdots \mathrm{I}$ interaction is predominantly dispersive and with a total energy component of $\sim-10 \mathrm{~kJ} / \mathrm{mol}$.

The characteristic close-packed molecular arrangement and isotropic distribution of interactions of comparable strength account for the brittleness in the crystals of OXH and OXF. Close-packed assemblies of OXH and OXF have face-to-face $\pi$-stacking and $\mathrm{C}-\mathrm{H} \cdots \pi$ interactions as the dominant interaction types. OXC and OXB have two-dimensional EFs (Fig. S28), with relatively weak halogen $\cdots$ halogen interactions describing the weakest plane. In the OXC, the Type-I $\left(\theta_{1}=\theta_{2}=161^{\circ}\right) \mathrm{Cl} \cdots \mathrm{Cl}$ interaction is more extended (by $\sim 0.1 \AA$ ) than the sum of the van der Waals radii; the short contact could be described as the crystal packing effect rather than with a chemical connotation. The absence of reliable interactions enables the adjacent layers to slip with respect to one another. The Type-I $\left(\theta_{1}=\theta_{2}=153^{\circ}\right) \mathrm{Br} \cdots \mathrm{Br}$ interactions have a distance $(d)$ shorter than the sum of the van der Waals radii by $0.2 \AA$; they are relatively weak and of dispersion characteristics. Of note, the computed EF shows no apparent evidence for the $\mathrm{Br} \cdots \mathrm{Br}$ interactions; instead, the neighboring layers connect through

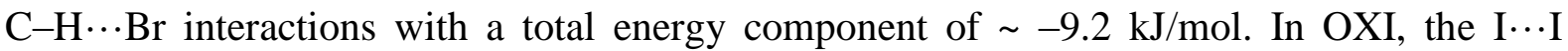
interaction is quasi Type-II $\left(\theta_{1}=176^{\circ}\right.$ and $\left.\theta_{2}=124^{\circ}\right)$ and is predominantly dispersive; the bond (4.04 $\AA$ ) is slightly longer than the sum of the van der Waals radii (3.96 $)$ ). The EF shows a near isotropic distribution of interactions in the OXI, but the interlayer region is prominently held through dispersive interactions. Thus, in $\mathrm{OXC}, \mathrm{OXB}$, and $\mathrm{OXI}$, the two-dimensional EF results from the robust interactions established by the oxalate and aromatic $\mathrm{C}-\mathrm{H}$ moieties within a layer and the weak dispersive halogen interactions in the interlayer region. Further, the calculated normalized contacts $(N c)$ values of the halogen interactions in the interlayer region are close to or higher than unity, which signifies their weak characteristics.

\begin{tabular}{cccc}
\hline Compound & $\boldsymbol{d}$ & $\boldsymbol{V r}$ & $\boldsymbol{N} \boldsymbol{c}$ \\
\hline BZF & & 2.94 & \\
BZC & 3.366 & 3.5 & 0.961714 \\
BZB & 3.845 & 3.7 & 1.039189 \\
BZI & 3.96 & 3.96 & 1 \\
\hline \multicolumn{5}{c}{ OXC } & 3.587 & 3.5 & 1.024857 \\
OXB & 3.51 & 3.7 & 0.948649 \\
OXI & 4.037 & 3.96 & 1.019444 \\
\hline \multicolumn{4}{c}{$d=$ bond distance; $V \mathrm{r}=$ sum of van der Waals radii; $N c=$ normalized contacts }
\end{tabular}


OXH

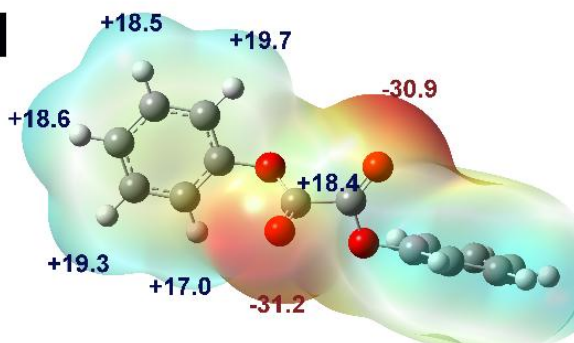

OXF

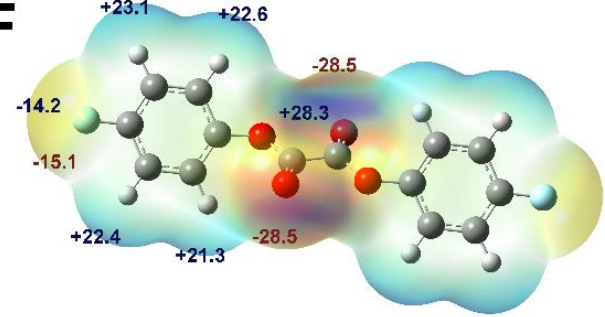

OXC

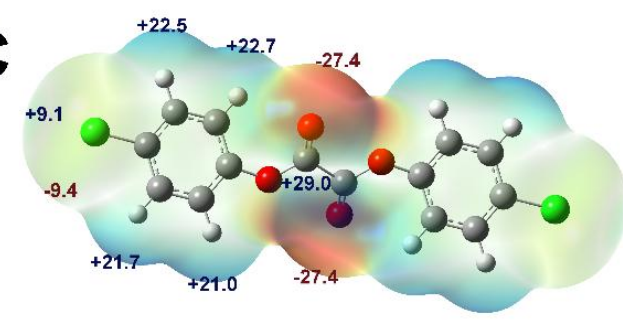

OXB

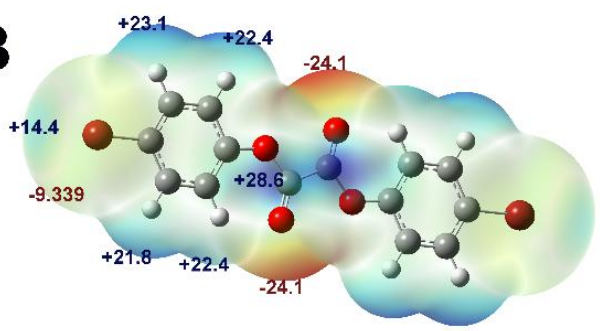

OXI

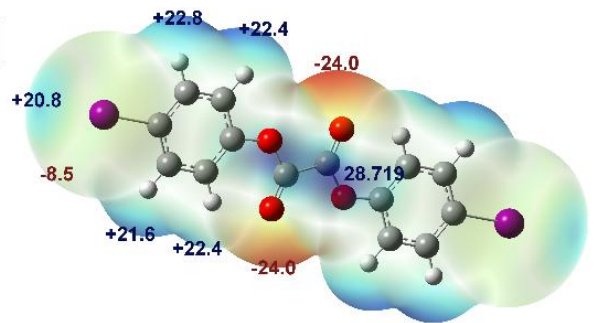

BZH

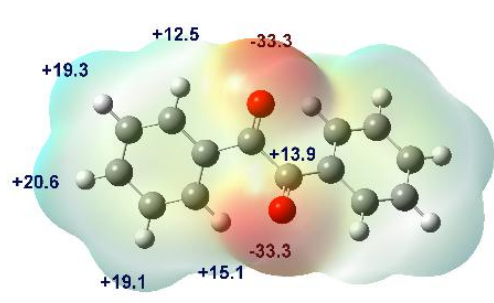

BZF

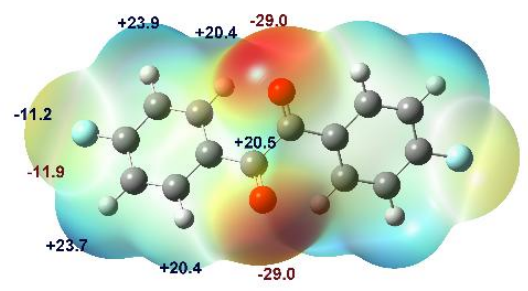

BZC

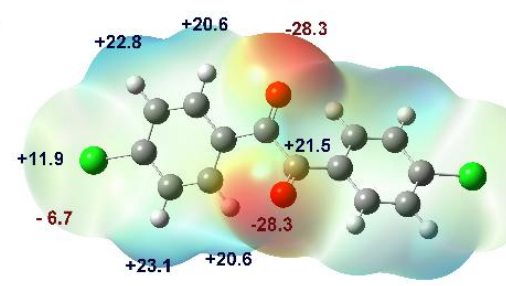

BZB

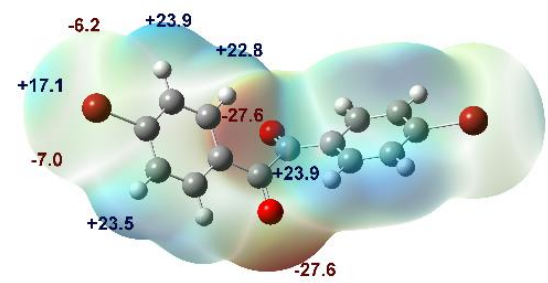

BZI

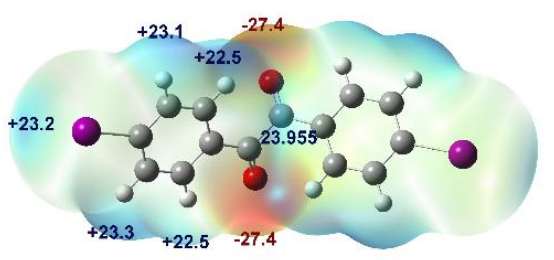

Fig. 8 MESP surfaces of the oxalate and benzil derivatives at the B3LYP/6$311+\mathrm{G}(\mathrm{d}, \mathrm{p}) / \mathrm{DGDZVP}$ level of theory. Isosurface 0.001 a.u. The MESP maximum and minimum energies are indicated in $\mathrm{kcal} \mathrm{mol}^{-1}$.

Molecular Electrostatic Potential (MESP) Analysis: Non-covalent interactions such as halogen bond, tetrel bond, chalcogen bond, and pnicogen bonds have their strength proportional to the size and characteristics of the " $\sigma$-hole or $\pi$-hole." ${ }^{42,43} \mathrm{We}$ used the computed MESP surfaces of the compounds to investigate the existence and intensity of the $\sigma$-holes and 
$\pi$-holes for the rational understanding of the interactions in the benzil and oxalate compounds. Because the strength of the interactions generally correlates with the magnitudes of the positive and negative electrostatic potentials, the sites of minima and maxima on the MESP can provide insights into the nature of non-covalent interactions. ${ }^{42,43}$

As demonstrated in Fig. 8, the most prominent negative MESPs are confined to the sites around the central oxalate or diketo moieties. At the same time, the aromatic $\mathrm{H}$-atoms account for high positive MESPs. Down the halogen series, the $\sigma$-hole trait and dimension increase, though they are less prominent vis-à-vis classic XB forming compounds such as tetrafluoro1,4-diiodobenzene or 1,4-dibromo tetrafluorobenzene. ${ }^{44,45}$ Of note, the positive region on the MESPs corresponding to the halogen $\sigma$-holes is less significant than on the aromatic $\mathrm{C}-\mathrm{H}$ regions. The lower magnitude of the positive MESPs corroborates the weak attributes of the halogen $\cdots$ halogen interactions observed in the structures. The central oxalate moiety with positive MESPs validates the $\mathrm{C}=\mathrm{O} \cdots \mathrm{C} \pi$-hole interactions in the crystal structures. Nevertheless, in benzil structures, the lower magnitude of the positive MESPs in the diketo moiety and its twisted conformations deter the formation of $\mathrm{C}=\mathrm{O} \cdots \mathrm{C}$ bonds. In a unique derivative, the magnitude of the MESP maxima at the halogen polar region is higher for the benzil compounds than its corresponding oxalate analog. In OXF, the $\mathrm{C}-\mathrm{F} \cdots \mathrm{C} \pi$-hole interaction also can be understood based on the complementarity of MESP charges.

Reduced Density Gradient (RDG) and Non-covalent Interaction (NCI) plot: We further used the RDG method to account for non-covalent interactions, particularly the possible $\mathrm{X} \cdots \mathrm{X}$ and $\mathrm{C}-\mathrm{H} \cdots \mathrm{X}$ interactions in the interlayer region. ${ }^{46,47}$ We plotted the RDG scatter plots and noncovalent interaction (NCI) plots to illustrate the interactions of interest (Fig. S18-S25). The isosurfaces are colored according to the values of $\operatorname{sign}\left(\lambda_{2}\right)$ r (a.u.), from $\AA 0.03$ to 0.02 a.u: blue represents robust, attractive interactions, green indicates van der Waals interactions, and red indicates repulsive/steric interactions. The analysis of the structures corroborates the MESP analysis and validates the weak nature of the interactions, especially the $\mathrm{X} \cdots \mathrm{X}$ interactions in the interlayer region (Fig. 9). The $\mathrm{X} \cdots \mathrm{X}$ interactions, as revealed by the NCI plot index analysis, show isosurfaces located between the halogen atoms. In both $\mathrm{OXC}$ and $\mathrm{BZC}$, the $\mathrm{C}-$ $\mathrm{Cl} \cdots \mathrm{Cl}-\mathrm{C}$ interactions are Type-I, making it chemically less significant. The $\mathrm{C}-\mathrm{Cl} \cdots \mathrm{Cl}-\mathrm{C}$ Type-I intermolecular topology is described by an irregular RDG isosurface between them. These isosurfaces correspond to the broad green in the $\operatorname{sign}\left(\lambda_{2}\right)<0$ regions of the $\rho \times \operatorname{sign}\left(\lambda_{2}\right)$ vs. RDG plot in $2 \mathrm{D}$, where $\lambda_{2}$ is the second eigenvalue of the Hessian of the charge density matrix. In both cases, the compounds show weak van der Waals interactions (>-0.01 a.u.); the energy difference between the two systems is negligible. Nevertheless, in BZC and BZB, the 
$\mathrm{C}-\mathrm{H} \cdots \mathrm{X}$ interactions play a complementary role to strengthen the interlayer region and implied by the corresponding isosurfaces located between the $\mathrm{CH}$ and halogen atoms. The broad red spike in the $\operatorname{sign}\left(\lambda_{2}\right)>0$ regions, indicative of steric repulsion, corresponds to an RDG domain appearing at the center of the phenyl rings. The other oxalate and benzil series members exhibit similar RDG scatter plots, indicating similar interaction characteristics. The isosurface becomes increasingly prominent in a unique series while moving down the halogen group. Thus in the I-derivatives, both benzil and oxalate compounds show comparatively prominent isosurface corresponding to the I $\cdots I$ interactions, though the RDG scatterplot identifies them as weak.

The MESP and the RDG scatterplot highlight the critical role of the interlayer region sustained through weak dispersive $\mathrm{X} \cdots \mathrm{X}$ bonds or their combination with $\mathrm{C}-\mathrm{H} \cdots \mathrm{X}$ interactions in determining the macromechanical properties. During elastic bending, the gridlike network sustained through weak dispersive $\mathrm{C}-\mathrm{H} \cdots \mathrm{O}$ and $\mathrm{C}-\mathrm{H} \cdots \pi$ interactions within the layers acts as a 'structural buffer.' With considerable tolerance towards the breaking and making upon bending/release of the external stress, weak dispersive interactions is critical in attaining the elastic bending behavior. It provides a spring effect, permitting regular structural changes at the bent site. The interactions in the outer arc elongate to accommodate the applied strain, whereas those in the inner arc get compressed (Scheme 2). The moderately weak and dispersive, but significant, $\mathrm{X} \cdots \mathrm{X}$ and $\mathrm{C}-\mathrm{H} \cdots \mathrm{X}$ interactions in the interlayer region provide resistance against long-range molecular displacements, thus allowing for the elastic deformation. In $\mathrm{BZB}$, the Type-II $\mathrm{Br} \cdots \mathrm{Br}$ and $\mathrm{C}-\mathrm{H} \cdots \mathrm{Br}$ interactions can operate at longer distances and keep the system in the attractive regime. The Type-I interactions in the Clderivative are further complemented by $\mathrm{C}-\mathrm{H} \cdots \mathrm{Cl}$ interactions, providing a stabilizing force to bring the system to the thermodynamic equilibrium. Thus, dispersive interactions in the interlayer region provide enough flexibility and impetus to the system to adopt partial molecular movements enabling it to restore the original state with the withdrawal of the applied stress. The inclined orientation of the molecules with respect to the surface also contributes to the minimal molecular movement. The near isotropic distribution of interactions allows them to have a greater elastic regime but beyond which the layers slip, thereby ensuing a plastic character. Considering the halogen-substituted benzil compounds, the elastic strain $\varepsilon$ in the order $\mathrm{Br}>\mathrm{Cl}>\mathrm{F}$ infers a better elastic property for the BZB, vis-à-vis BZF. Nevertheless, the elastoplastic charectaristics manifested as $\varepsilon_{\text {(stage-II) }}$ in the order $\mathrm{F}>\mathrm{Cl}>\mathrm{Br}$, reflects a better elastoplastic property in the BZF (Table S3). 


\section{BZF}
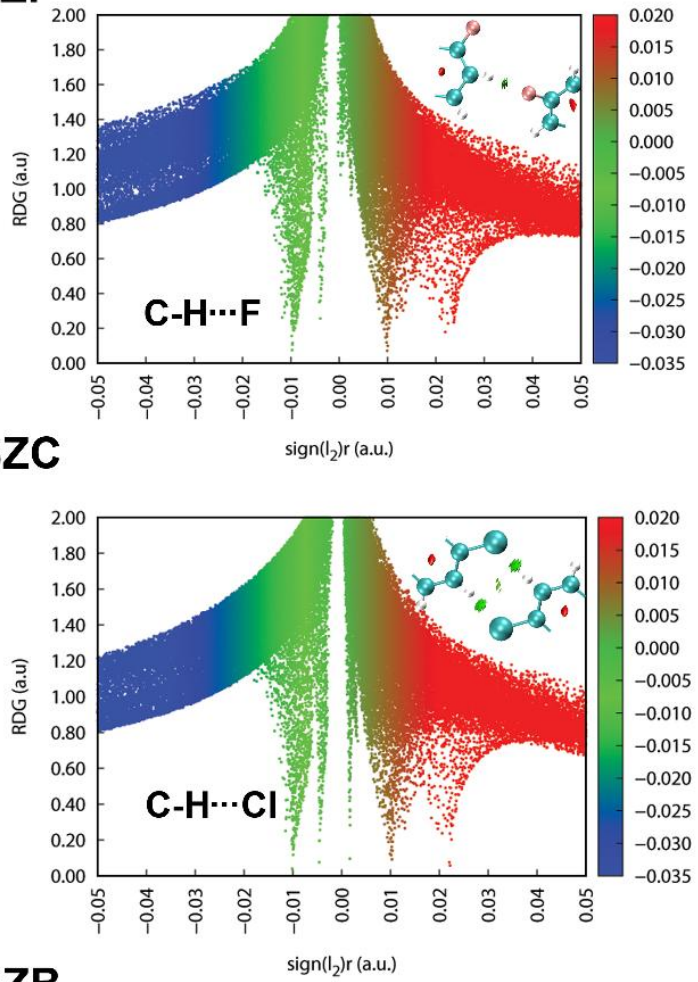

BZB

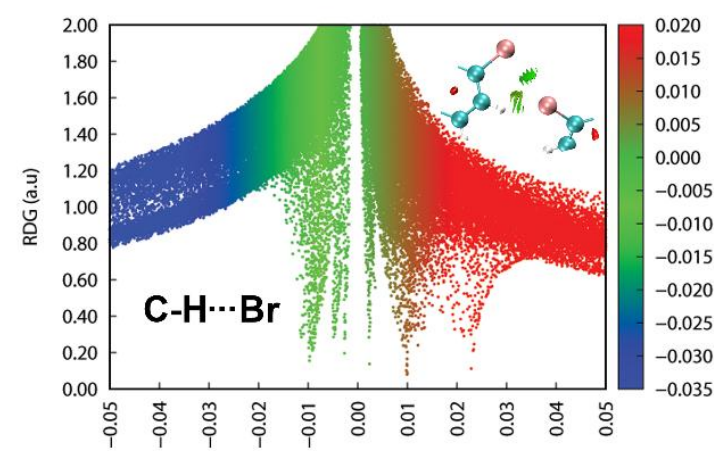

BZI

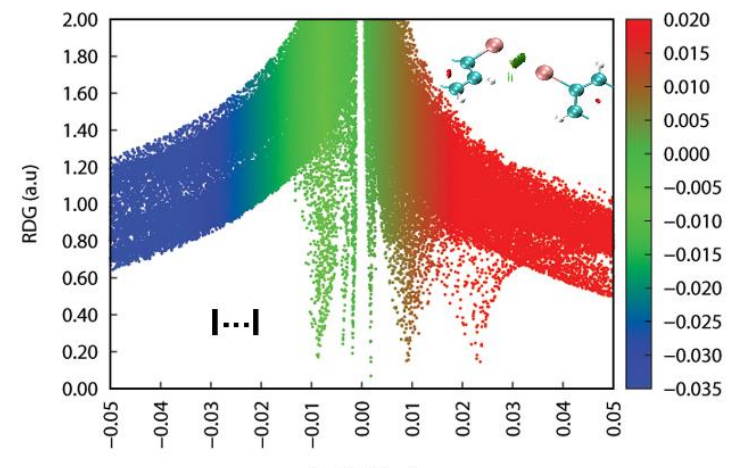

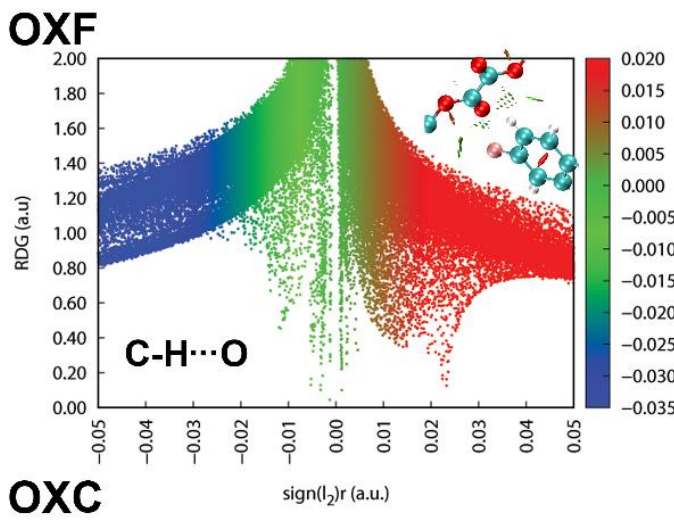

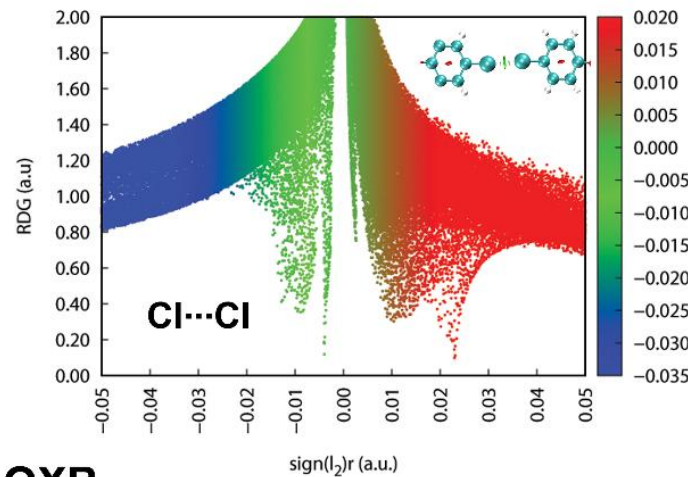

\section{OXB}

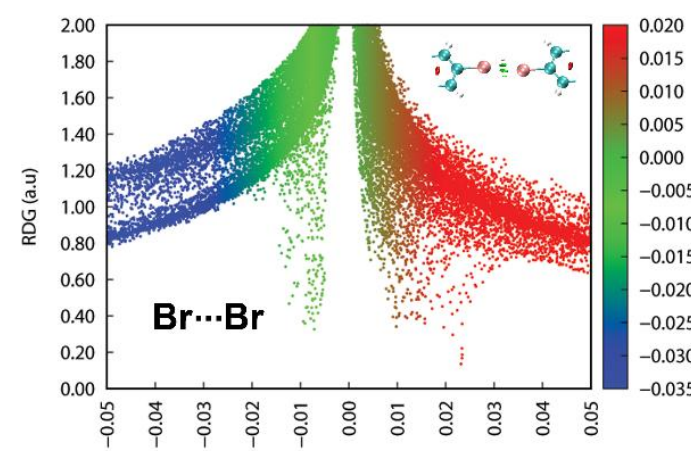

OXI

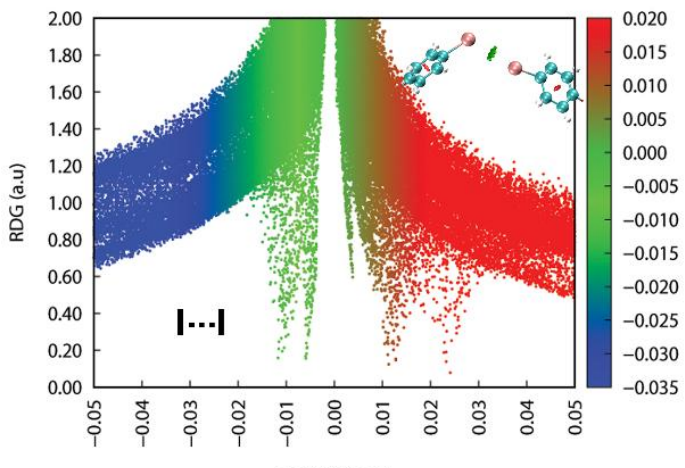

$\operatorname{sign}\left(\mathrm{I}_{2}\right) \mathrm{r}$ (a.u.)

Fig. 9 Scatter plot of reduced density gradient (RDG) against the sign of second Hessian eigenvalue times the electron density. The corresponding RDG electron density surface plots for the benzil and oxalate compounds are also provided. 


\section{Structure-mechanical property correlations}

Oxalate crystals also exhibit the property of the co-existence of elastic and plastic deformation, though the elastic domain is considerably lesser vis-à-vis the benzil compounds. In the oxalate compounds (OXC, OXB, and $\mathrm{OXI})$, though the molecules have similar structural topology, the contributing interaction types differ, and so do their mechanical properties. The non-covalent bonds within the layer are a combination of $\pi \cdots \pi, \mathrm{C}=\mathrm{O} \cdots \mathrm{C}(=\mathrm{O})$ and $\mathrm{C}-\mathrm{H} \cdots \mathrm{O}$ interactions, having both the electrostatic and dispersive components; the interactions, hence are more robust within the layer. However, in the interlayer region, the $\mathrm{X} \cdots \mathrm{X}$ interactions are either Type-I or longer than the sum of van der Waals radii making them relatively weak. The $\pi$-hole carbonyl-carbonyl interactions induce added degree of anisotropy in the system, even though the structure lacks any classical electrostatic interactions. Further, the layer separation about the slip regions in the oxalates is slightly bigger (OXB: $2.34 \AA$ and OXC: $2.11 \AA$ ) than in the benzil series (BZB: $2.25 \AA$; BZC: $2.03 \AA$, and BZF: $1.79 \AA$ ). Consequently, the oxalate crystals exhibit significant anisotropy in the structure, making the layer slipping more energetically viable and favoring the plastic deformation.

(a)

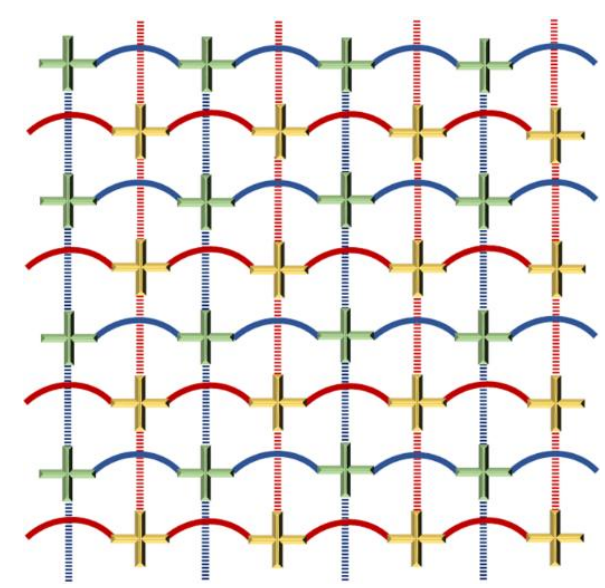

(b)

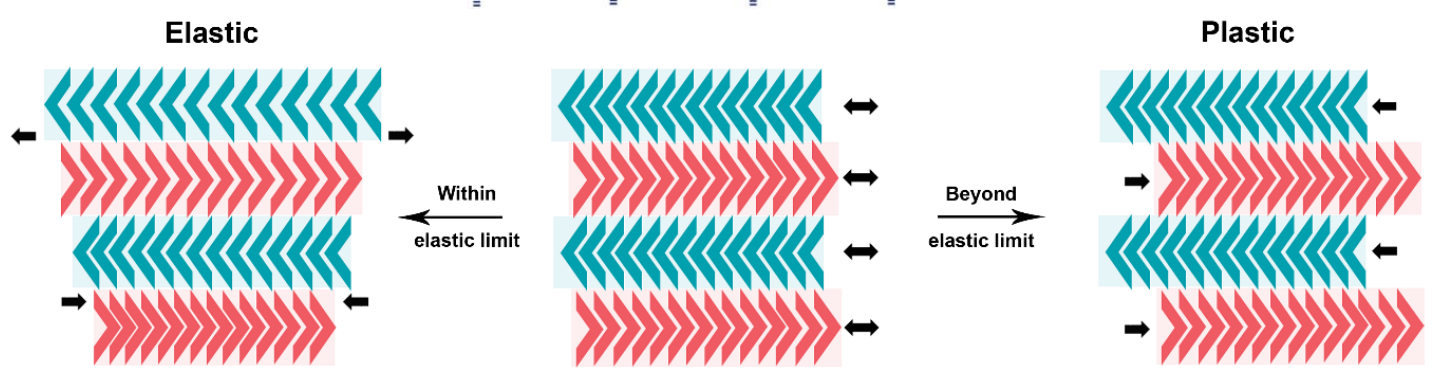

Scheme 2 Schematic representation of (a) the grid-like network formed in the benzil structure; (b) the proposed layer transformation that leads to elastic and plastic defromation in the crystals.

In general, plastic deformation in molecular crystals is due to either slip or twinning. The benzil compounds have a stacked-layered arrangement with weak interactions in the interlayer region. However, in the absence of classic electrostatic interactions, the observed 
anisotropy is not large enough to induce extensive plastic characteristics to the crystals. Thinner benzil crystals exhibit better elastic flexibility compared to their thicker counterparts. The observation corroborates some of the earlier reports that highlighted the elastic property of thinner crystals compared to the thicker ones. ${ }^{31}$ Such size-dependent mechanical response could be correlated with possible defects also. With a relatively lesser number of defects than the thicker ones, the mechanical properties of the thinner crystals could be the manifestation of the intrinsic structural factors. In thicker crystals, the defect concentration and their mobility contribute significantly to the mechanical response. ${ }^{48,49}$ The hypothesis conforms with an earlier observation that the crystals in the nanometer regime have significantly higher stiffness $(\sim 85 \%)$ compared to the millimeter size crystals. ${ }^{50}$

(a)
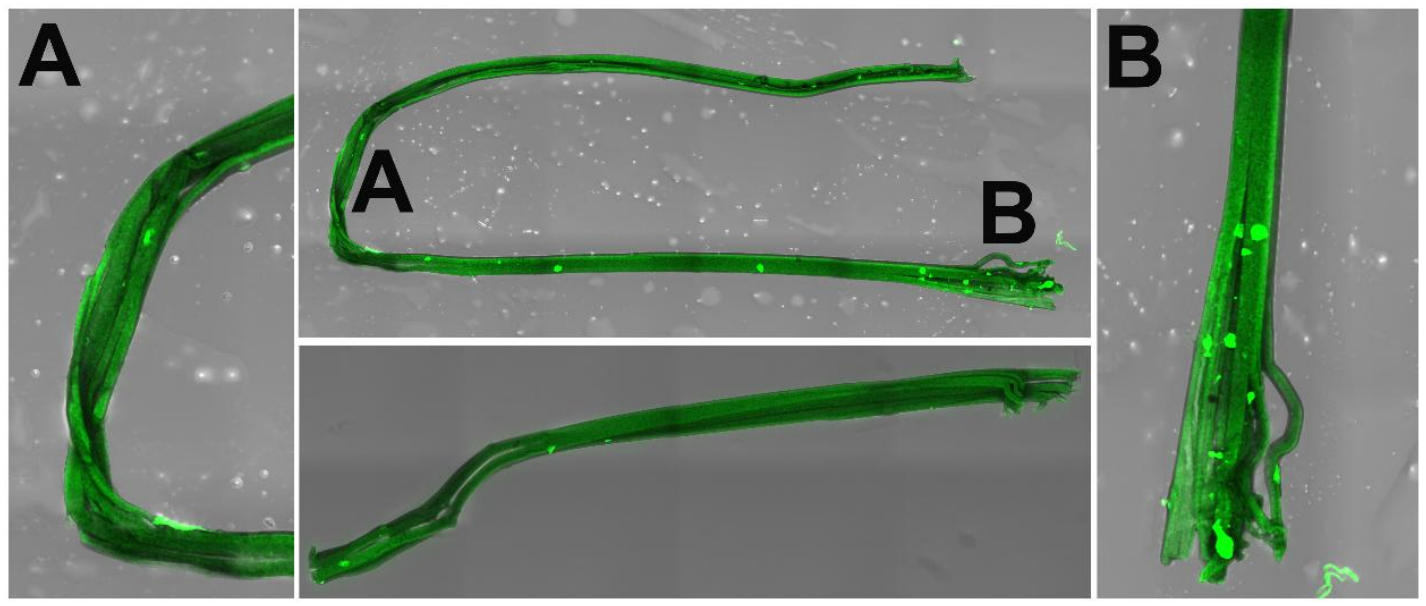

(b)

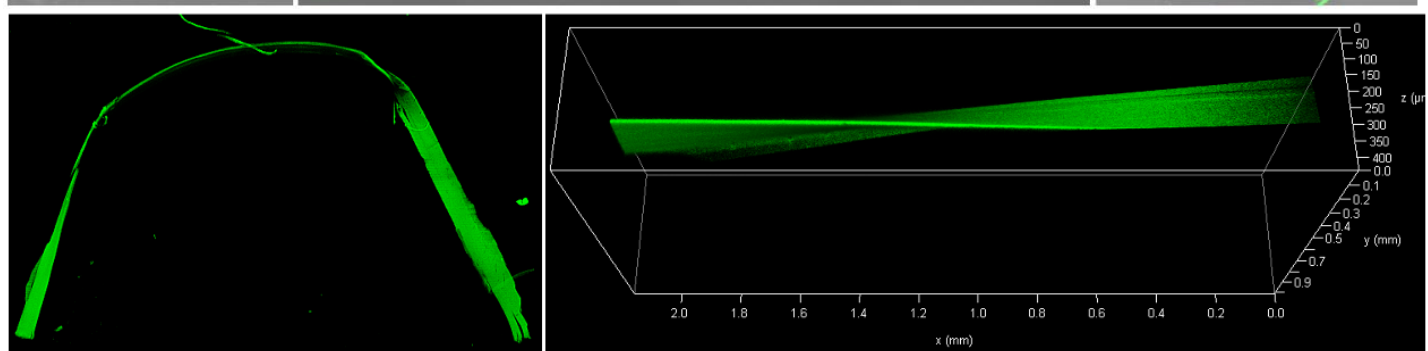

Fig. 10 The confocal microscopy images. (a) the bent crystal of BZC. Note the delamination observed in the area corresponding the ends and the bent region. (b) The bending and twisting observed in BZF.

Upon cutting or smudging with a blunt substrate, the ends of the long tape-like crystals of BZF, BZC, and BZB exfoliate into bundles of fibrils (Fig. 10,11 and S11). The fluorescent images of the crystals evident the characteristic observation. The resultant fibrils exhibit high flexibility. We further analyzed the macroscopic crystal delamination property in the benzil crystals using confocal microscopy. The crystals of BZC show excellent plastic deformation under applied stress. The confocal microscope images ratify the delamination of the crystals into thin fibrils at the bent region and the ends (Fig. 10a). Due to the inherent anisotropy, 
molecular crystals could show various imperfections when bending strain is applied in different directions and delaminate. Such crystal delamination could be a critical parameter in ensuing plasticity in the benzil crystals. The plane with the least attachment energy provides an energetically viable route to delaminate under applied bending strain. For example, in BZC, (002) containing the interplanar region sustained through weak $\mathrm{Cl} \cdots \mathrm{Cl}$ interactions could give in under bending strain. Thus, apart from the microscopic structural contribution, crystal delamination could be considered a possible macroscopic factor, contributing to attaining the plastic deformation at the higher applied stress. (Fig. 10a; inset A). Within the elastic limit, the microscopic structural elements that favor elastic deformation dominate the deformation mechanism in the crystals. At higher stress, further to the long-range molecular movements (microscopic factors), defect formation and propagation, as well as macroscopic factors such as crystal delamination, contribute to irreversible deformation in crystals. In oxalate systems, however, crystal delamination is not extensive. Hence the observed plastic deformation in the oxalate crystals could be understood in terms of structural anisotropy, with a limited macroscopic contribution.

(a)
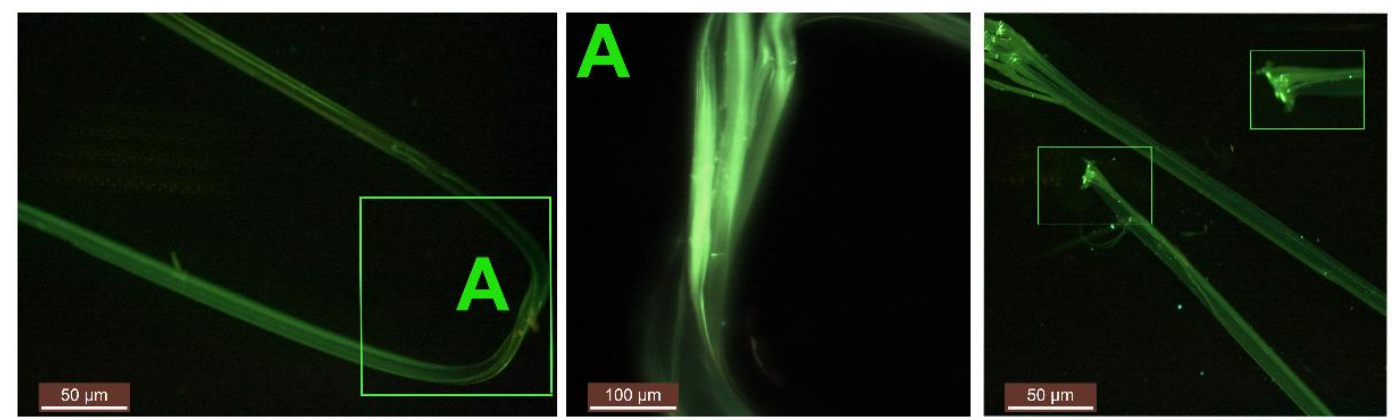

(b)

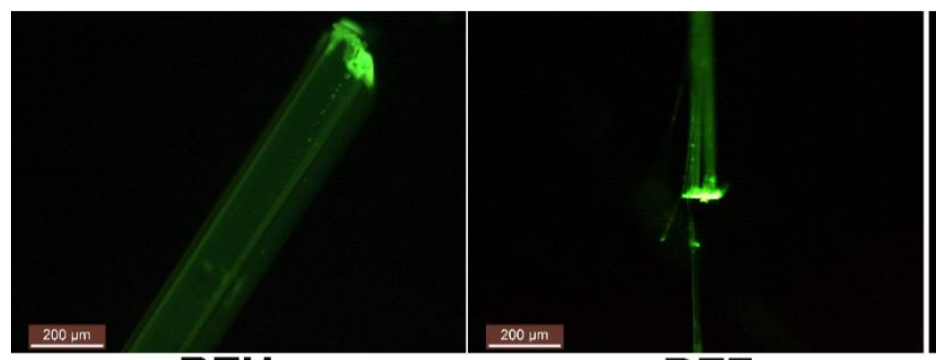

BZH
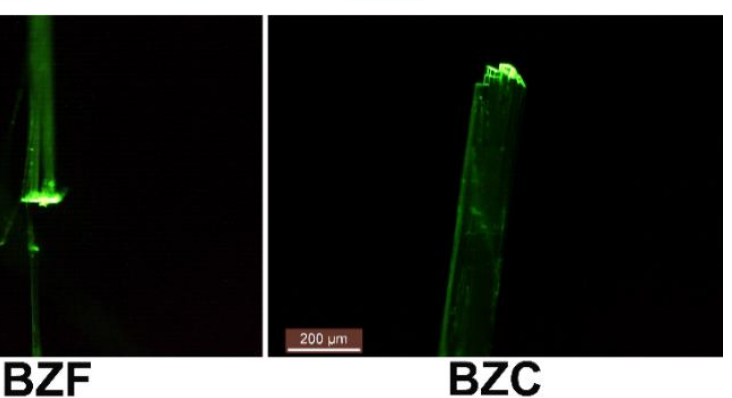

BZC

Fig. 11 The fluorescent microscopy images of the benzil crystals. (a) highlights the delamination behavior in the BZC crystals. (b) shows the higher emission intensities at the ends of the crystals than the central parts, suggesting a conventional photowaveguide effect.

The benzil compounds have interesting emission properties: besides their intense green emission characteristics, they exhibit crystallization induced phosphorescence (CIP). ${ }^{51}$ Accordingly, BZH, BZF, and BZB emit at 521, 500, and $526 \mathrm{~nm}$, respectively, upon excited at $365 \mathrm{~nm}$. Of note, the dimorphs of the F-derivative (BZF_O and BZF) exhibit distinct emission 
spectra (Fig. S9b). The benzil crystals (BZF, BZC, and BZB) because of the isostructural characteristics, the recorded absorption and emission spectra are comparable (Fig. S9). A recent report highlights the waveguiding properties of $\mathrm{BZB} .{ }^{31} \mathrm{We}$ noted that in all the members of the benzil series (BZH, BZF, BZC, and BZB), both ends of the crystals show higher emission intensities than the central parts, suggesting a conventional photowaveguide effect (Fig. 11). While various derivatives of benzil compounds, including BZB, are known to exhibit roomtemperature phosphorescence (RTP), BZC and BZF are also likely to show the effect. ${ }^{51,52}$ However, we lack access to facilities that could quantify the RTP and the waveguiding properties of these crystals. Mechanically exfoliated thin fibrils also fluoresce intensely at their ends Fig. 11. Long crystal edges look similar to a bundle of optical fibers under UV irradiation. Deformed crystals in the bent state show appreciable intense emission at the crystal ends (Fig. S10, S11 and S12). The crystals delaminated by excessive bending still retain the property. Thus, combining the mechanical flexibility in tandem with photonic traits in organic crystals could give enormous opportunity for fabricating miniature organic optoelectronic materials. ${ }^{53,54}$

\section{Conclusions}

In the preceding sections, we have demonstrated the co-existence of the elastic and plastic deformation in the molecular crystals by taking halogen-substituted benzil compounds as typical examples. Though the studies related to elastic and plastic crystals are known, the intermediate type examples that display elasticity and plasticity under different stress settings are exceptional. The elastic nature of the benzil crystals can be correlated to the isotropic deployment of the interactions in the interlayer and intralayer regions, which provide an initial resistance to the slippage of layers. The criss-cross square-grid network in the benzil compounds provides the required rigidity to the overall structure without breaking. At the same time, the inclined orientation of the molecules and the dispersive halogen interactions that stitch the layer structure provide enough flexibility and impetus to the system to adopt partial molecular movements and restore the original state with the withdrawal of the applied stress. Thus, the unique structural features observed in the benzil compounds are crucial to enabling the co-existence of 'continuity' and 'deformation' synergically under external stimuli. In the intermediate stress, the crystals of benzil compounds show elastoplastic characteristics, which is rare in molecular crystals. At higher stress, further to the long-range molecular movements (microscopic factors), defect formation and propagation, as well as macroscopic factors such as crystal delamination, contribute to irreversible deformation in crystals. Though with a 
comparable stacked-layered arrangement, the contributing interaction types in oxalates differ, and so do their mechanical properties. A combination of $\pi \cdots \pi, \mathrm{C}=\mathrm{O} \cdots \mathrm{C}(=\mathrm{O})$ and $\mathrm{C}-\mathrm{H} \cdots \mathrm{O}$ interactions, having both the electrostatic and dispersive components add more robustness to the interactions within a layer. In contrast, the $\mathrm{X} \cdots \mathrm{X}$ interactions (that are either Type-I or longer than the sum of van der Waals radii) that sustains the interlayer region allow long-range slippage of layers. Thus, replacing the twisted diketo moiety in benzil with a rigid and coplanar central oxalate moiety enabled an enhanced contribution of $\mathrm{C}=\mathrm{O} \cdots \mathrm{C}$ interactions, furthering the anisotropic element in the crystal packing. The nature and strength of the interactions formed by the halogen substituents in the interlayer region and tuning the distribution of dispersive interactions are vital in attaining the delicate balance of interactions and their macroscopic mechanical properties. The work highlights the structural basis for the coexistence of elastic and plastic bending characteristics in molecular crystals and the possibility to fine-tune the extend of anisotropy to modulate the macroscopic mechanical response. In the absence of a universal supramolecular strategy to improve upon a specific mechanical property of a unique crystalline material, we need to analyze an extensive library of materials with comparable contributing factors to derive better correlations between the structures and properties.

\section{References}

1. H. Jiang and W. Hu, Angew. Chem. Int. Ed., 2020, 59, 1408.

2. T. Someya, Z. Bao, and G. G. Malliaras, Nature, 2016, 540, 379.

3. P. Naumov, S. Chizhik, M. K. Panda, N. K. Nath, and E. Boldyreva, Chem. Rev., 2015, 115, 12440 .

4. K. Kinbara and T. Aida, Chem. Rev., 2005, 105, 1377.

5. S. Varughese, M. S. R. N. Kiran, K. A. Solanko, A. D. Bond, U. Ramamurty, and G. R. Desiraju, Chem. Sci., 2011, 2, 2236.

6. B. P. A. Gabriele, C. J. Williams, M. E. Lauer, B. Derby, and A. J. Cruz-Cabeza, CrystEngComm, 2021, 23, 5826.

7. M. Reichel, D. Dosch, T. Klapötke, and K. Karaghiosoff, J. Am. Chem. Soc., 2019, 141, 19911.

8. C. Zhang, X. Xue, Y. Cao, Y. Zhou, H. Li, J. Zhou, and T. Gao, CrystEngComm, 2013, 15, 6837. 
9. A. J. Thompson, A. Worthy, A. Grosjean, J. R. Price, J. C. McMurtrie, and J. K. Clegg, CrystEngComm, 2021, 23, 5731.

10. X. Chu, Z. Lu, B. Tang, B. Liu, K. Ye, and H. Zhang, J. Phys. Chem. Lett., 2020, 11, 5433.

11. T. Aida and E. W. Meijer, Isr. J. Chem., 2020, 60, 33.

12. R. Geitner, F.-B. Legesse, N. Kuhl, T. W. Bocklitz, S. Zechel, J. Vitz, M. Hager, U. S. Schubert, B. Dietzek, M. Schmitt, and J. Popp, Chem. Eur. J., 2018, 24, 2493.

13. M. Pisacic, I. Biljan, I. Kodrin, N. Popov, Z. Soldin, and M. Dakovic, Chem. Mater., $2021,33,3660$.

14. S. Varughese, M. S. R. N. Kiran, U. Ramamurty, and G. R. Desiraju, Angew. Chem. Int. Ed., 2013, 52, 2701.

15. P. Naumov, D. P. Karothu, E. Ahmed, L. Catalano, P. Commins, J. Mahmoud Halabi, M. B. Al-Handawi, and L. Li, J. Am. Chem. Soc., 2020, 142, 13256.

16. A. Hasija and D. Chopra, CrystEngComm, 2021, 23, 5711.

17. S. Varughese, M. S. R. N. Kiran, U. Ramamurty, and G. R. Desiraju, Chem. Asian J., 2012, 7, 2118.

18. E. Ahmed, D. P. Karothu, and P. Naumov, Angew. Chem. Int. Ed., 2018, 57, 8837.

19. M. K. Mishra, S. Varughese, U. Ramamurty, and G. R. Desiraju, J. Am. Chem. Soc. 2013, 135, 8121.

20. A. J. Thompson, A. I. Chamorro Orué, A. J. Nair, J. R. Price, J. McMurtrie, and J. K. Clegg, Chem. Soc. Rev., 2021, 50, 11725.

21. B. Tang, B. Liu, H. Liu, and H. Zhang, Adv. Funct. Mater., 2020, 30, 2004116.

22. C. M. Reddy, R. C. Gundakaram, S. Basavoju, M. T. Kirchner, K. A. Padmanabhan, and G. R. Desiraju, Chem. Commun., 20053945.

23. K. Zhang, C. C. Sun, Y. Liu, C. Wang, P. Shi, J. Xu, S. Wu, and J. Gong, Chem. Mater., 2021, 33, 1053.

24. F. Liu, D. E. Hooks, N. Li, N. A. Mara, and J. A. Swift, Chem. Mater., 2018, 30, 3798.

25. T. Sasaki and S. Takamizawa, Cryst. Growth Des., 2020, 20, 6990.

26. S. Kusumoto, A. Saso, H. Ohmagari, M. Hasegawa, Y. Kim, M. Nakamura, L. F. Lindoy, and S. Hayami, ChemPlusChem, 2020, 85, 1692.

27. S. P. Thomas, M. W. Shi, G. A. Koutsantonis, D. Jayatilaka, A. J. Edwards, and M. A. Spackman, Angew. Chem. Int. Ed., 2017, 56, 8468.

28. S. Saha and G. R. Desiraju, Chem. Commun., 2016, 52, 7676. 
29. S. Li and D. Yan, ACS Appl. Mater. Inter., 2018, 10, 22703.

30. S. SeethaLekshmi, M. S. R. N. Kiran, U. Ramamurty, and S. Varughese, Chem. Eur. J., 2019, 25, 526.

31. H. Liu, Z. Bian, Q. Cheng, L. Lan, Y. Wang, and H. Zhang, Chem. Sci., 2019, 10, 227.

32. K. Naim, M. Singh, S. Sharma, R. V. Nair, P. Venugopalan, S. C. Sahoo, and P. P. Neelakandan, Chem. Eur. J., 2020, 26, 11979.

33. S. Das, S. Saha, M. Sahu, A. Mondal, and C. M. Reddy, Angew. Chem. Int. Ed., 2021, n/a, e202115359.

34. M. Felsmann, F. Eissmann, A. Schwarzer, and E. Weber, Cryst. Growth Des., 2011, 11, 982.

35. D. E. Wu, Y. H. Luo, M. N. Wang, Q. L. Liu, G. J. Wen, L. J. Zhu, C. P. Fan, and B. W. Sun, Struct. Chem., 2017, 28, 1731.

36. J. J. McKinnon, D. Jayatilaka, and M. A. Spackman, Chem. Commun., 20073814.

37. F. H. Allen, C. A. Baalham, J. P. M. Lommerse, and P. R. Raithby, Acta Crystallogr., Sect.B, 1998, 54, 320.

38. B. Sahariah and B. K. Sarma, Chem. Sci., 2019, 10, 909.

39. A. Rahim, P. Saha, K. K. Jha, N. Sukumar, and B. K. Sarma, Nature Commun., 2017, 8, 78.

40. M. J. Turner, S. P. Thomas, M. W. Shi, D. Jayatilaka, and M. A. Spackman, Chem. Commun., 2015, 51, 3735.

41. S. Saha and G. R. Desiraju, Chem. Commun., 2017, 53, 6371.

42. P. Politzer, J. S. Murray, and T. Clark, Phys. Chem. Chem. Phys., 2013, 15, 11178.

43. J. S. Murray, P. Lane, T. Clark, K. E. Riley, and P. Politzer, J. Mol. Model., 2012, 18, 541.

44. Y. Zhao, Y. Cotelle, N. Sakai, and S. Matile, J. Am. Chem. Soc., 2016, 138, 4270.

45. G. Cavallo, P. Metrangolo, R. Milani, T. Pilati, A. Priimagi, G. Resnati, and G. Terraneo, Chem. Rev., 2016, 116, 2478.

46. E. R. Johnson, S. Keinan, P. Mori-Sánchez, J. Contreras-García, A. J. Cohen, and W. Yang, J. Am. Chem. Soc., 2010, 132, 6498.

47. G. Saleh, C. Gatti, and L. Lo Presti, Comput. Theor. Chem., 2012, 998, 148.

48. S. Bhandary, A. J. Thompson, J. C. McMurtrie, J. K. Clegg, P. Ghosh, S. R. N. K. Mangalampalli, S. Takamizawa, and D. Chopra, Chem. Commun., 2020, 56, 12841. 
49. M. Li, C. Zhang, M. Li, F. Liu, L. Zhou, Z. Gao, J. Sun, D. Han, and J. Gong, Chem. Eng. J., 2022, 429, 132450(1).

50. C. Karunatilaka, D. K. Bucar, L. R. Ditzler, T. Friscic, D. C. Swenson, L. R. MacGillivray, and A. V. Tivanski, Angew. Chem. Int. Ed., 2011, 50, 8642.

51. Y. Y. Gong, Y. Tan, H. Li, Y. Zhang, W. Z. Yuan, Y. M. Zhang, J. Z. Sun, and B. Z. Tang, Sci. China Chem., 2013, 56, 1183.

52. S. Yamada, T. Higashida, Y. Wang, M. Morita, T. Hosokai, K. Maduwantha, K. R. Koswattage, and T. Konno, Beilstein J. Org. Chem., 2020, 16, 1154.

53. A. L. Briseno, R. J. Tseng, M. M. Ling, E. H. L. Falcao, Y. Yang, F. Wudl, and Z. Bao, Adv. Mater., 2006, 18, 2320.

54. E. Ahmed, A. L. Briseno, Y. Xia, and S. A. Jenekhe, J. Am. Chem. Soc., 2008, 130, 1118. 
Flexible organic crystals. Understanding the tractable co-existence of elastic and plastic bending

Indira S. Divya, Saravanan Kandasamy, Krzysztof Woźniak* and Sunil Varughese*

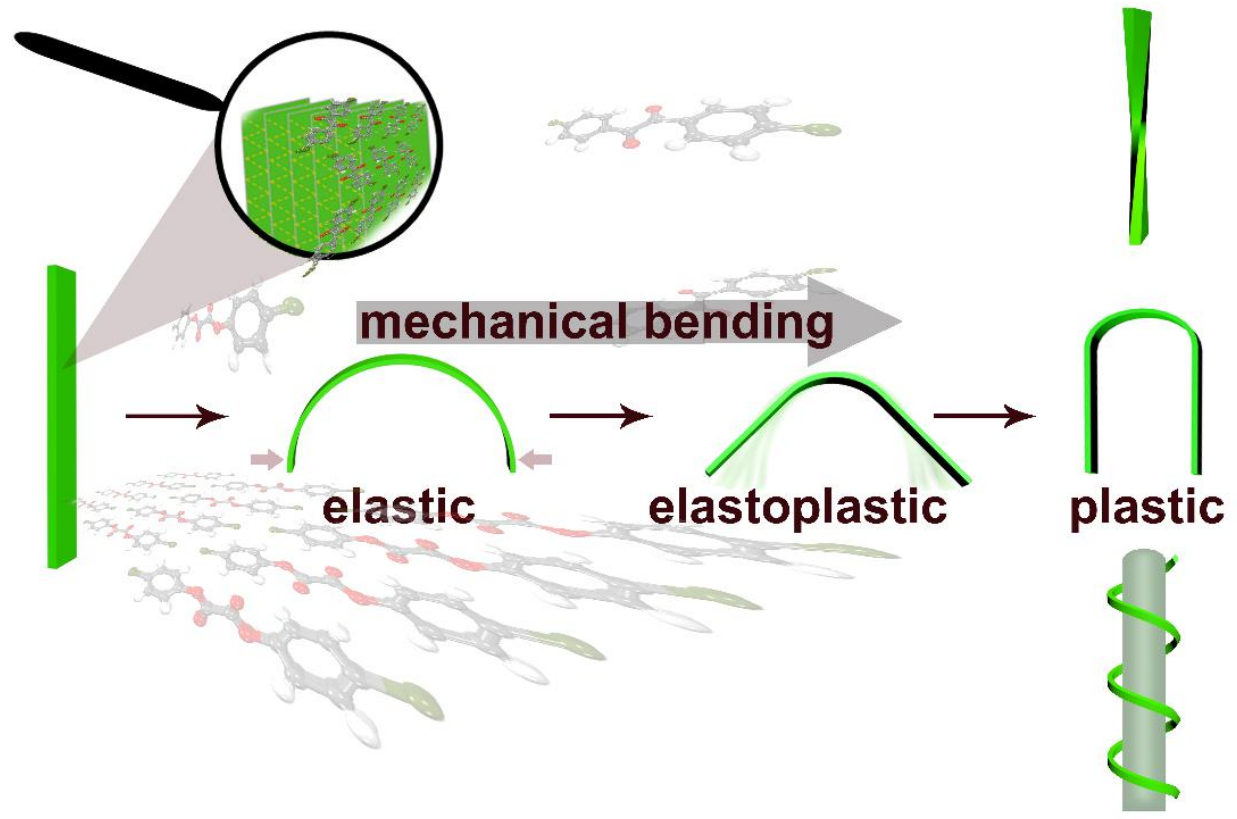

